\title{
Customer Lifetime Value Models \\ Do they predict actual behaviour?
}

Master's Thesis

Glenn Cooksley

Principal Supervisor: Associate Professor Malcolm Wright December 2007 


\section{Acknowledgements}

What sculpture is to a block of marble, education is to a human soul.

Joseph Addison (1672 - 1719)

Completing this thesis has been challenging and highly rewarding. I wish to pass on my appreciation to my supervisor Malcolm for his patience and guidance.

Most importantly, this thesis has not been possible without my family. To Cath and Sol - your love and support over this year reminds me how lucky I am to have you in my life.

Glenn 


\section{Abstract}

Accurate input information is the cornerstone of sound managerial decision making. Assessing the future lifetime value of customers is a key component in making accurate managerial decisions such as how to apply scarce organisation resources on retention or acquisition activities (Blattberg and Deighton, 1996). Additionally, accurate customer lifetime value (CLV) calculation can be used for effective segmentation of customers.

Berger and Nasr (1998) recognised the need for an improved approach to customer lifetime valuation calculation. The model proposed by Berger and Nasr (1998) differed from historical approaches, such as the Recency, Frequency, and Monetary (RFM) method, by predicting the future state of existing customers and discounting the projected cash flow over time. Whilst the RFM model was popular as noted by Reinartz and Kumar, (2000), it was limited in accurately calculating the future value of a group of customers and was applied in segmentation classification.

Berger and Nasr's (1998) model found favour in literature where subsequent contributions followed in areas; Managerial application of the model findings, alternative approaches to calculating the model inputs, and introducing alternative variables or techniques in the CLV calculation model itself.

The literature confirmed Berger and Nasr's (1998) approach as suitable for examination in this study however also revealed a general lack of empirical validation 
for Berger and Nasr's (1998). A review of literature detailed several extensions to the theory and modelling literature on CLV and several propositions relating to this area of theory development. These were contributions mostly conceptual by nature and few supported their concepts with empirical validation.

This empirical study provides an important contribution by examining the predictive accuracy of Berger and Nasr's (1998) CLV calculation model. The purpose of this research was to compare Berger and Nasr's (1998) CLV model's prediction of customer lifetime value against the actual value data over a specific period for a set cohort of residential segment consumers from a leading New Zealand energy retailer. This study goes further to examine the sensitivity of the model's calculation output to a change in input variables.

The findings of this research challenge the predictive accuracy of Berger and Nasr's (1998) CLV model. The model was applied using both large (total cohort) and small (segments) customer groups to understand how what level of accuracy can be achieved in different contexts.

The study identified a number of limitations such as the use of a constant retention rate, and not adequately accommodating the level of customer heterogeneity. The sensitivity of the model to change in the input variables supported Gupta, Lehmann and Stuart's (2004) research showing the retention variable was the critical input as it was the most influential on the model calculation. The marketing and discount rate variables had little to no influence on the model calculation outcome. Several 
propositions identified in literature on this subject were examined with many supported such as Reichheld and Sasser's (1990) observation that businesses lose 15\% - 20\% of their customers each year. Wyner's (1999) proposition was also supported in that the cohort when segmented demonstrated considerable different characteristics including patterns of attrition.

This research presents empirical findings that will assist further theory development in the area of accurate measurement of Customer Lifetime Value (CLV) and promotes further examination of Berger and Nasr's (1998) CLV model. 


\section{Table of contents}

Page

1 Introduction 1

2 Literature review 4

2.1 Recency, Frequency and Monetary (RFM) methodology 5

$\begin{array}{lll}2.2 & \text { Zero defections } & 7\end{array}$

$\begin{array}{lll}2.3 & \text { CLV model foundations } & 9\end{array}$

$\begin{array}{lll}2.4 & \text { Customer migration } & 11\end{array}$

$\begin{array}{lll}2.5 & \text { Non-mathematical inputs } & 12\end{array}$

$\begin{array}{lll}\text { 2.6 CLV calculation - the discounted cash flow approach } & 14\end{array}$

$\begin{array}{lll}2.7 & \text { Progressing CLV model calculation } & 17\end{array}$

$\begin{array}{lll}2.8 & \text { Option theory } & 18\end{array}$

$\begin{array}{ll}2.9 \text { In the microscope } & 20\end{array}$

2.10 Customer retention calculation $\quad 22$

2.11 CLV and customer equity 30

2.12 A product perspective $\quad 32$

2.13 The context of risk 33

2.14 Literature review - summary 36

$3 \quad$ Research question $\quad 39$

$\begin{array}{lll}3.1 & \text { Principal research question } & 39\end{array}$

3.2 Related propositions for examination $\quad 40$

$4 \quad$ Methodology 43

4.1 Research design 44

$\begin{array}{lll}4.2 & \text { Data } & 45\end{array}$ 
$\begin{array}{lll}4.3 & \text { Limitations } & 46\end{array}$

4.3.1 Marketing spend $\quad 46$

$\begin{array}{lll}4.3 .2 \text { Costs } & 47\end{array}$

$\begin{array}{lll}4.3 .3 & \text { Value } & 47\end{array}$

$\begin{array}{lll}\text { 4.3.4 Customer tenure } & 48\end{array}$

$\begin{array}{lll}5 & \text { Results and discussion } & 49\end{array}$

$\begin{array}{lll}5.1 & \text { Introduction } & 49\end{array}$

5.2 Cohort description 50

5.2.1 General market conditions $\quad 50$

5.2.2 Retention $\quad 51$

$\begin{array}{ll}5.2 .3 \text { Consumption } & 55\end{array}$

5.3 Application of the CLV model - appropriate fit 58

5.3.1 Input variables $\quad 58$

5.4 Model calculation compared to actual state $\quad 60$

$\begin{array}{lll}5.5 & \text { Sensitivity } & 62\end{array}$

5.5.1 Retention 63

$\begin{array}{ll}\text { 5.5.2 Marketing spend } & 65\end{array}$

$\begin{array}{lll}\text { 5.5.3 Discount rate } & 66\end{array}$

$\begin{array}{lll}5.6 & \text { Summary - model calculation } & 67\end{array}$

$\begin{array}{lll}\text { 5.6.1 Accuracy of findings } & 67\end{array}$

5.6.2 Sensitivity - retention rate $\quad 68$

5.6.3 Sensitivity - marketing spend and discount rate $\quad 68$

$\begin{array}{lll}5.7 & \text { Segment analysis } & 69\end{array}$

$\begin{array}{lll}\text { 5.7.1 Lost customers } & 70\end{array}$ 
$\begin{array}{ll}\text { 5.7.2 Consumption profile } & 71\end{array}$

$\begin{array}{ll}\text { 5.7.3 Consumption per customer } & 73\end{array}$

$\begin{array}{ll}\text { 5.7.4 Comparison - ad hoc segmentation } & 75\end{array}$

$\begin{array}{lll}\text { 5.7.5 Segment profile } 2006 & 78\end{array}$

5.7.5.1 Light segment $2003 \quad 79$

5.7.5.2 Medium segment $2003 \quad 80$

5.7.5.3 Heavy segment 2003

5.7.6 Re-testing for fit and sensitivity 82

5.7.7 Is hindsight perfect vision? 84

5.7.8 Propensity to repurchase (retain) 85

$\begin{array}{lll}5.8 & \text { Contribution } & 86\end{array}$

6 Conclusion $\quad 88$

$\begin{array}{lll}6.1 & \text { Summary of findings } & 91\end{array}$

$\begin{array}{lll}6.2 & \text { Related observations } & 98\end{array}$

6.2.1 The RFM approach 98

$\begin{array}{lll}\text { 6.2.2 Retention } & 99\end{array}$

$\begin{array}{lll}7 & \text { Limitations and future research } & 101\end{array}$

$\begin{array}{lll}7.1 & \text { Limitations } & 101\end{array}$

$\begin{array}{lll}7.2 & \text { Future research } & 102\end{array}$

$8 \quad$ References 106 


\section{List of tables}

Page

Table 1: Customer attrition by year 53

Table 2: Cumulative customer attrition $\quad 54$

Table 3: Cohort profile (total customer base) 56

Table 4: Model vs. actual comparison 61

Table 5: Sensitivity analysis on the retention (rtn) input variable 63

Table 6: Sensitivity analysis on the marketing (M) input variable 65

Table 7: Sensitivity analysis on the discount rate (d) input variable 67

Table 8: Percentage of customers lost each year $\quad 71$

Table 9: Total cohort and segment profile 75

Table 10: Light segment of 2003 profiled in $2006 \quad 79$

Table 11: Medium segment of 2003 profiled in 2006

Table 12: Heavy segment of 2003 profiled in 2006

Table 13: Fit of model to actual - total cohort and segments 82

Table 14: Re-calculation based on using actual average defection rate 84 


\section{List of figures}

Page

Figure 1: CLV model by Berger and Nasr (1998) 15

Figure 2: Markov Chain Model (MCM) calculation 23

Figure 3: CLV model by Rust et al. (2004) 28

Figure 4: Number of surviving customers per annum 52

Figure 5: Number of customers per annum in each segment (2004 classification) 72

Figure 6: Proportional makeup of customer numbers by segment 73

Figure 7: Average consumption per customer per annum (rolling reclassification) 74

Figure 8: Comparing customer number by segment in 2003 and $2006 \quad 76$

Figure 9: Comparing average consumption by segment in 2003 and $2006 \quad 76$ 


\section{Introduction}

A key component of success in marketing is accurate identification and retention of the right customer (Blattberg and Deighton, 1991). This is the basis of the modern marketing paradigm in relationship marketing. The value of retained loyal customers is observed by McKenna (1993) as a source of competitive advantage.

Business is often about compromise. In the context of acquiring and retaining customers, organisations have limited resources and need effective tools to make the best decision possible. Compromise comes from balancing the use of scarce resources such as how much to invest in retention or acquisition activity (or whether to at all). Segmentation is a common tool used for efficient use of resources and to improve the outcome of targeted marketing activity. There are several approaches used to group customers into segments such as geographical, consumption, and value. However, in recent times academics and practitioners have investigated ways to segment customers using the classification of the lifetime value of the customers to an organisation.

Customer value is a central tenet of customer relationship marketing (Gronroos, 1991; Morgan and Hunt, 1994; and Wyner, 1996). The inherent risk of basing decisions on the future sate of customer value is reliance on the accuracy of the value calculation method employed. Berger and Nasr (1998) set out to assist managers in that decision making by providing a Customer Lifetime Value (CLV) calculation model. The outputs of this calculation are designed to assist managers in efficient resource allocation decisions and focus acquisition and retention strategies. The concern of 
many authors such as Blattberg, Getz, and Thomas, (2001), Gupta and Lehmann, (2005), and Rust, Lemon and Zeithaml, (2004) is the risk of an inaccurate calculation of future value will lead to poor options taking. The error in making business decisions based on unreliable or inaccurate information presents academics and practitioners strong motivation to develop trusted accurate models to calculate the value of customers.

The progression of CLV calculation models in academic literature has been built on the work by Berger and Nasr (1998). The subsequent literature in this area has been predominantly conceptual and focused on how input variables of the CLV model are established or how managers should interpret results. The calculation of future value incorporates the widely accepted method of discounted cash flow. Despite a considerable body of work on CLV calculation models, a concerning observation is the lack of empirical testing and model validation. Contributing an empirical study to the evaluation of a well established customer lifetime value calculation model is the role of this research.

The first step in the examination of CLV modelling was selection of a suitable CLV calculation model for evaluation. Following identification of the right model, it is applied to actual customer data recruited from a commercial energy retail organisation in New Zealand. This organisation (working title - Rata Energy) consented to providing a cohort of residential profile consumers with monthly recorded consumption data over the years 2003 to 2006. In addition to the data extract received, 
management at Rata Energy were interviewed to provide any necessary inputs for the CLV calculation, specifically marketing spend and the discount rate used in the model.

The research initially undertook descriptive statistical analysis of the cohort, profiling the customer group, identifying the retention rate for each year of the cohort study. The selected CLV model is then applied using inputs from the initial year of the cohort (2004) to predict the value of those customers in 2006 . The findings are compared to a net present value calculation, of the actual consumption information for those same customers over the same period. Analysis of the results is undertaken and discussed in relation to the implication on the research problem and relevant propositions identified in literature. Following establishment of the relative accuracy of 'fit' of the model the sensitivity of inputs is investigated. This examines the sensitivity of the CLV model outcome to change in the input variables.

The issue of customer heterogeneity is also examined. The cohort is segmented by volume consumption and then the analysis based on these segment specific input variables is revisited. The purpose is to test if using granular level inputs will lead to a more accurate 'fit' of the model.

The findings have been shared with Rata Energy as a part of this research project. Early feedback acknowledged the need to review and refine current approaches to customer segmentation and value calculation. Additionally it has led to further discussion on strategy development and investment decisions for these and broader customer groups. 


\section{Literature Review}

The discipline of marketing has progressed in recent times where organisations' aim to become 'customer focused' and shift from transactional interactions to building long term relationships with customers. This is predicated on the belief that long term customer relationships relate to higher profitability (Reichheld and Sasser, 1990). This research does not directly challenge this premise and assumes this as the operating tenet of marketing for this research context.

The shift to a customer orientation necessitates the development of tools to enable marketers to effectively identify and benefit from highly profitable customers. One essential aspect of operating under this orientation is the need for accurate customer lifetime value measurement. To effectively and accurately project the profitability of a customer (or segment) is vital to an organisation. Understanding lifetime value of a firm's customer base will contribute to efficiency in making informed decisions on utilisation of scarce resources, and to understand the marginal returns to marketing execution (Blattberg and Deighton, 1996; Levitt, 1986; McKenna, 1991; Webster, 1994; Dickson, 1997; Kotler, 1997). Specifically, so organisations can target the right customers based on the value of those customers to an organisation i.e. the tenet of the direct marketing approach. "Attracting and keeping the highest value customers is the cornerstone of a successful marketing program" (Blattberg and Deighton, 1996, p.136). 
The following literature review highlights practices used to calculate the value of customers of the period of time they have a purchase relationship with a company and discusses the implications on managerial decision making. The literature on customer lifetime value has several themes. It commences with how the frame work of RFM (Recency, Frequency and Monetary) can enable decision making to alternative customer lifetime value calculation models, with several models and variations proposed. It also extends to how to apply the findings of these various calculation processes to business decision making. This literature review profiles key models and variations introduced. The aim of the literature review, in addition to providing sound background on rationale and model progression, was the identification of a foundation customer lifetime value calculation model to use in this examination.

The discussion on CLV modelling begins by profiling a widely adopted model used by marketing managers to optimise resource allocation called the Recency, Frequency and Monetary model (RFM).

\subsection{Recency, Frequency and Monetary (RFM) methodology}

The RFM approach was introduced by Cullinan (1978) who was credited with identifying the three variables. The model was later extended by Bauer (1988) to apply to managerial decision making. The adoption and popularity of this model is based on the relative ease of use, the accessibility of the inputs that can be sourced using an organisations own transaction data. Lastly, the relatively straight-forward logic has strong application appeal. Stone (1995) proposed using the RFM 
methodology as an approach to assist in targeting valued customers by placing weightings on purchases to rank customers.

RFM analysis profiles customers by the three variables of purchase behaviour, which is how recently the customer has purchased (recency), how often they purchase (frequency), and the level of customer spending (monetary value) (Cullinan, 1978). Combining the size of the purchase with frequency allows for customer segmentation (Colombo and Jiang, 1999, and Shepherd, 1990). The recency element allows organisations to understand the potential loyalty of a customer relative to their expected purchase frequency or previous purchase behaviour. Interpretations are made from the analysis that customers who purchase frequently are more likely to purchase again. Lastly, customers who spend more, and make regular purchases, are more likely to continue spending more and think more favourably of the brand.

The common application of the RFM approach is to segment customers based on observation in literature (Colombo and Jiang, 1999, Shepherd, 1990, and Reinartz and Kumar, 2000). This has found favour by practitioners in its application to direct marketing and database marketing disciplines. The use of RFM is however not without criticism. This is due to several limitations of the model that lead to poor decision-making (Miglautsch, 2002). The ability of the RFM method to assist in effective resource allocation is also challenged by Reinartz and Kumar (2000) who observed "the use of the RFM model can result in suboptimal allocation of limited resources" (Reinartz and Kumar, 2000, p.18). The RFM approach is limited in application in managerial strategy development as it does not factor for time in relation 
to cash flow over the period of the customer tenure. Additionally, it fails to consider the opportunity to increase retention rate thereby improving profitability. These limitations reduce the contribution this approach can make to informed strategic decision making.

\subsection{Zero defections}

Another early attempt for measuring customer value came out of services marketing. Reichheld and Sasser (1990) commented that a need to retain customers is vital to increased value and hence proposed organisations pursue 'zero defects'. Reichheld and Sasser (1990) made several observations about customer value and the reduction of defection rates. "Reducing defections by just $5 \%$ generated $85 \%$ more profits in one bank's branch system, 50\% more in an insurance brokerage, and 30\% more in an autoservice chain" (Reichheld and Sasser, 1990, p.107).

Reichheld and Sasser (1990) also discussed how tenure relates to customer profitability. "Companies with loyal long time customers can financially outperform competitors with lower unit costs and high market share but high customer churn" (Reichheld and Sasser, 1990, p.108). They also made an observation of the relatively consistent level of defection in business. "It is common for a business to lose $15 \%$ to $20 \%$ of its customers each year" (Reichheld and Sasser, 1990, p.108). The excerpts above present interesting and challenging propositions relevant to this research. 
A subsequent article by Reichheld (1996) presented research findings that "On average, the CEO's of USA corporations lose half their customers every five years" (Reichheld, 1996, p.56).

A justification for retention investment was also proposed by Reichheld (1996). The impact of customer defection on profitability was discussed with commentary on several reasons why keeping 5\% more customers will increase a firms profit by $100 \%$. "Older customers tend to produce greater cash flow and profits than newer ones" (Reichheld, 1996, p.56).

Reichheld and Sasser (1990) and Reichheld (1996) both presented interesting propositions albeit in somewhat sensationalised headlines. Headline statements of $100 \%$ more profit from $5 \%$ more retention do not necessarily reflect the averages of Reichheld (1996) and Reichheld and Sasser's (1990) findings. They reported increases of $85 \%$ in banking, $50 \%$ insurance and $30 \%$ in an auto service chain. These findings were not empirically based and both articles lead to questioning the integrity of the statements presented because of the variation between the headlines and the actual data provided in these articles.

Irrespective of the integrity of the data and degree of affect reported, Reichheld and Sasser (1990) were successful in providing a catalyst to subsequent papers and general interest in this area. This was particularly relevant to the area of Customer Lifetime Values (CLV) in business, how that value may be calculated, and the link to decision making for resource allocation through to marketing strategy development. 


\subsection{CLV model foundations}

Several conceptual papers discussed the concepts of customer value and customer equity, highlighting issues with factors influencing the realisation of that value or equity. In 1996 Blattberg and Deighton introduced a paper that discussed the important role Customer Lifetime Value (CLV) calculation models could play for organisations in making informed and profitable decisions in regard to maximising equity of the customer and firm. Blattberg and Deighton's (1996) paper was key at a time when academics and practitioners had adopted the paradigm of the relationship marketing concept and were interested in the relationship between CLV calculations and business decision making.

Blattberg and Deighton's (1996) influential paper sparked discussion on methodology and application of CLV, and on the conceptual benefits of applying CLV calculations directly to business decisions. Blattberg and Deighton's (1996) conceptual paper introduced an approach for resource utility decision making to optimise acquisition and retention activities. The paper outlined a broad approach to the calculation of optimal spend on acquisition and retention spending and linked it to CLV modelling. The difference between the lifetime value of a customer and customer equity was a distinction introduced by Blattberg and Deighton (1996) and continued to be an area of subsequent literature discussion. This early focus did not address the calculation directly though had introduced a rationale. It provided insight into why the requirement for reliable, accurate models of calculation of customer lifetime value would continue to grow. 
Berger and Nasr-Bechwati (2001) addressed the issue of customer equity with a paper that discussed the influence of promotional budgets. They introduced a decision calculus conceptual model in which manager judgements would be incorporated into calculations. The article discussed the subsequent impact on acquisition and retention options as well as specific media category expenditure.

Van Raaij (2005) presented a rationale for the use of CLV calculation tools in business, emphasising the role customer profitability analysis had in managerial strategic decision making and marketing planning. The paper introduced a conceptual model founded on a cost model orientation and set about to provide understanding of how profitability was distributed throughout a customer base.

The relationship between CLV and shareholder value was addressed by Berger, Eechambadi, George, Lehmann, Rizley and Venkatesan (2006) introducing a chain of effects framework to explain the dynamics of the relationship. They introduced the concept of a range of steps that preceded CLV calculation and consideration of the competitive environment in how it impacts on the equity of the customer. They also introduced the direct relationship CLV modelling can have in calculating the value of a firm at the shareholder level.

In addition to discussing the relationship between CLV, customer equity and shareholder value a relevant stream of literature acknowledge the role customer valuation has in calculating outcomes of customer migration. 


\subsection{Customer migration}

Dwyer (1997) introduced a taxonomy of the Lifetime Value (LTV) for buyer-seller relationships that could be employed to formulate a calculation of the lifetime value and measure migration behaviours to a population. This contribution is similar in ways to the introduction of option theory proposed by Levett, Page, Nel, Pitt, Berthon, and Money (1999). Dwyer (1997) applied the taxonomy to two distinct groups of customers defined by Jackson (1985) as the lost-for-good customer scenario where a customer makes a long term commitment to an organisation and the always-a-share customer scenario where the firm is prepared to give any vendor a portion of their business. These customer groups were formed due to their relationship type and commitment to a supplier. The model relies on purchase recency to predict repeat purchase behaviour. The purchase propensity based on previous behaviours establishes a propensity estimate called the recency cell in the model. This was a positive contribution as it sought to project future states through introduction of a propensity element to retention.

The main focus of Dwyer's (1997) article was on managerial decision-making and implications for designing and budgeting for customer acquisition programs, specifically in relation to migration situations. Whilst introducing the propensity element the article did not directly contribute to CLV computation methods. The literature in this area was generally conceptual and outlined the benefits to organisations who adopt suitable CLV calculation approaches. The following 
discussion moves from managerial implications to exploring the calculation process in the context of considering non-mathematical inputs into the CLV calculation.

\subsection{Non-mathematical inputs}

Berger, Weinberg and Hanna (2003) applied a CLV model to highlight how decision making can be influenced by specific migration models when based on real data. The CLV model was used to measure the retention of cruise liner ticket purchase customers. They acknowledged in their paper that it would have been very unlikely that any mathematical model could capture all the inputs needed. Introducing nonmathematical subjective elements to CLV calculations presents a challenge to get a consistent accurate use of the inputs for the calculation. As well as accuracy the model must use inputs that are easy to source. Lastly, the model must be easy to use in order to gain practical adoption.

Helm (2003) introduced the concept of word-of-mouth as a major determinant of Customer Lifetime Value (CLV). Helm (2003) introduced Herrmann and Fuerderer's (1997) model that calculated CLV based on inputs such as referrals. The model proposed was highly complex including other variables such as price sensitivity, cross buy potential, referral behaviour and re-buying behaviour. The level of subjective inputs presents a challenge with Helm (2003) noting that many inputs needed an "educated guess" (Helm, 2003, p.132) in order for practitioners to attain all the required inputs to be effective in modelling. Whilst referrals may have a role for 
consumers in the decision making process, the context of high or low involvement transactions needs consideration.

Gruca and Rego (2005) investigated the concept of value by focusing on growth and stability as key characteristics of future cash flows. The article focused on the role satisfaction had on future cash flows. It presented findings at industry level using a USA national customer satisfaction database. Annualised profitability of firms in different sectors was used to illustrate a correlation between satisfaction and industry cash flow. This was at a high level and application to customers at firm level was an acknowledged area that required future research. Interestingly, the article reported that large firms were less efficient in increasing their cash flows yet did not provide insight into the potential drivers for this finding.

The literature at this point has mostly focused on the progression of theory on CLV from the conceptual rationale and early models, through to discussion on various nonmathematical inputs. In addition, the role CLV modelling has on business decision making for resource allocation has been highlighted. The literature shows a strong willingness and rationale for CLV calculation but lacked comprehensive empirical validation.

The focus shifts from the context of application to actual methods used for the calculation of lifetime value. The purpose of the remaining literature review is to highlight contributions in the area of CLV modelling. The literature relates to 
contributions of either core model designs or on ways to improve the input variables for the CLV calculation model.

\subsection{CLV calculation - the discounted cash flow approach}

Berger and Nasr (1998) presented a seminal paper specifically focused on a series of mathematical calculation models for determining Customer Lifetime Value (CLV). The CLV model uses the widely accepted Discounted Cash Flow (DCF) approach. Berger and Nasr (1998) note there are two steps being a need to "project the net cash flows that the firm expects to receive from the customer over time. Next, calculate the present value of that stream of cash flows" (Berger and Nasr, 1998, p.19)

The model suggests a three step CLV calculation process. It commenced with a calculation of the gross contribution discounted over time. Then established the amount of marketing expenditure discounted over time and then subtracted the marketing expense from the gross contribution to establish the lifetime value. The model is presented in Figure 1 and provides a detailed breakdown of the calculation approach. 
Figure 1: CLV model by Berger and Nasr (1998)

$$
\mathrm{CLV}=\left\{G C * \sum_{i=0}^{n}\left[r^{\mathrm{i}} /(1+d)^{i}\right]\right\}-\left\{M * \sum_{i=l}^{n}\left[r^{i-1} /(1+d)^{i-0.5}\right]\right\}
$$

(Berger and Nasr, 1998, p. 21)

Where:

$G C$ Is the (expected) yearly gross contribution margin per customer. It is equal to revenue minus cost of sales.

$M \quad$ Is the (relevant) promotion costs per customer per year. To reflect mid-year marketing expenditure it is possible to introduce 0.5 into the equation.

$i \quad$ Is the purchase cycle

$n \quad$ Is the length, in years, for the period over which cash flows are to be projected. Berger and Nasr (1998) noted that this period was likely to be highly dependent on the industry. Carpenter (1995) commented that extending the model further than five years involves too much guesswork in high tech industries (however, Berger and Nasr (1998) noted that longer periods may be plausible for durable products). There should also be consideration given to the contractual nature of the relationship with the customer. There are instances where 10 year contracts are put in place and hence the relative value of that relationship can be established with some accuracy and these calculations become pivotal to the initial negotiation strategy and process. 
$r \quad$ Is the yearly retention rate. This is the proportion of customers expected to continue buying the company's goods or services in the subsequent year. Note this application takes a 'customer group' level approach and the literature illustrated that this can be adapted to the individual level.

$d \quad$ Is the yearly discount rate (appropriate for marketing investments).

There are two notable exclusions from Berger and Nasr's (1998) approach to this model. They are the exclusion of costs for acquiring the customer and fixed cost components as inputs in the calculation. The rationale was that the model works to determine specifically the contribution margin of a customer. Consequently these were not included in the calculations, aligning with other direct marketing studies on the treatment of these variables. Berger and Nasr (1998) believed that this led their model to be appropriately conservative. The most challenging element of this CLV model acknowledged by Berger and Nasr (1998) was accurate projection of cash flows. This observation is consistent for all models in this area of focus.

The flexibility of Berger and Nasr's (1998) model was demonstrated through changing the characteristics of a number of input variables or introducing new variables to illustrate application to various 'real world' scenarios. The variables that were either changed or introduced were the length of projection period, frequency of sales, spend and rate of retention, amount of annual revenue, discrete vs. continuous cash flows, 
and lastly includes Dwyer's (1997) propensity projection of purchase recency. This diversity was illustrated however not empirically validated.

Dwyer's (1997) article was supportive of Berger and Nasr's (1998) CLV model illustrating in a conceptual paper a breadth of application and set up a road map of possible empirical validation under various scenarios before this model could be endorsed as a foundation formula. It is observed that despite the relatively limited empirical testing of Berger and Nasr's (1998) model, their approach had been widely adopted in literature.

Berger and Nasr's (1998) paper introduced a CLV model approach that has been widely adopted. This is evidenced with over two hundred and forty subsequent articles noted in a literature citation search on the Google Scholar website. This model became regarded as a foundation for CLV calculation modelling. Subsequent literature adopted the principles proposed by Berger and Nasr (1998) focusing on varying approaches to calculate model inputs as well as proposed new inputs.

\subsection{Progressing CLV model calculation}

Wyner's (1999) paper discussed the implications for management if organisations fail to take a segment orientated approach to viewing their customers and adopt the approach that all customers are treated as one group with similar needs and characteristics. Wyner (1999) observed that some customer segments exhibit different patterns of defection than others. This proposition presented important implications 
for much of the work to date on CLV as it had focused predominantly on looking at a total customer base perspective to equate the value to a firm of a customer base.

Mulhern (1999) did not contribute a change in the CLV calculation method. The article concentrated on the validity of several of the input variables to profitability analysis introducing Schmittlein, Morrison, and Colombo (1987) and Schmittlein and Peterson's (1994) technique of delineating the status of a customer to ensure they are 'active'.

The article by Mulhern (1999) supported the work done by Berger and Nasr (1998). It extended the CLV approach to consideration of a customer replacement costs element, and used a Tobin's $q$ ratio to reflect this cost. The introduction of the Tobin's $q$ ratio was noted by Hayashi (1982) as a preferred approach to account for the replacement cost of the customer in CLV calculation which is used in customer profitability analysis. This conceptually illustrated the structural customer profitability model for business to business transactions for a pharmaceutical manufacturer. The article illustrated the use of a CLV measurement in for the purpose of effectively segmenting customers. An alternative approach was introduced by Levett et al. (1999) that introduced a new approach to CLV calculation with the application of option theory.

\subsection{Option theory}

Levett, Page, Nel, Pitt, Berthon and Money (1999) presented an alternative approach to the calculation for Customer Lifetime Value (CLV) introducing option theory into the 
CLV calculation process. Levett et al. (1999) purport the use of their proposed CLV calculation approach would improve accuracy as it incorporated previous decisions as inputs to future decisions. This option valuation applies to customer re-purchase decision making. Option theory is used to capture the sequence of activities that happen when a customer is faced with a choice to purchase or not. This binary nature can be developed into a binomial lattice to better represent a probability element to valuing a customer "... appraises customers as a series of call options rather than a single series of expected profit contributions" (Levett et al. 1999, p.283). Levett et al. (1999) purported addition of option theory into the calculation of CLV would improve the accuracy of the model and produce results that better reflect a 'real world' context.

Levett et al. (1999) admit that the option valuation model is similar to Berger and Nasr's (1998) model in the way value is calculated. Levett et al. (1999) purport that the value of the option valuation model is the ability for managers to apply a context to a consumer's choice to undertake a transaction and not just to the total expected contribution as considered in Berger and Nasr's (1998) calculation.

Whilst an interesting contribution to the valuation of a customer, Levett et al.'s (1999) work received limited literature coverage and model extension in both academic and practical application. Levett et al. (1999) suggest there is benefit in further research "if previous purchases have a significant bearing on the probability of current purchases, in situations where the value of the customer is more sensitive to the level of probability than profitability growth" (Levett et al. 1999, p.283). 
Whilst option theory presents an interesting conceptual method in adding a level of probability the use of it addressed the context of dynamic consumer environments. This model is conceptual and is not widely applied by academics or practitioners.

\subsection{In the microscope}

An additional consideration in relation to Berger and Nasr's (1998) model was the inference that the model was best suited to application of contractual relationships between a customer and firm. This limitation was observed by Reinartz and Kumar (2000) who tested the model in the non-contractual context finding several limitations such as suitable consideration of the level of switching costs to the customer. If switching costs are low in the non-contractual setting, the customer switches easily. However, for long term relationships the customer needs to weigh up aspects such as the loss of loyalty schemes.

Reinartz and Kumar (2000) undertook one of the early empirical studies investigating four commonly held propositions associated with customer profitability over time. The research undertaken by Reinartz and Kumar (2000) used data taken from a USA catalogue retailer in the general merchandise category. The total number of observations, over the study period, came from 9,167 households made up of two cohorts - those that purchased over the 36 month period (total study period) and those that purchased over a 35 month period (cohort 2's $1^{\text {st }}$ purchase taking place one month after those in Cohort 1). Estimation of the parameters for the calculation used the approach suggested by Schmittlein, Morrison and Colombo's (1987) and subsequently 
Schmittlein and Peterson (1994). Taking the inputs from that calculation process Reinartz and Kumar (2000) applied the analysis to the Berger and Nasr's (1998) CLV calculation model.

The propositions and findings were:

1. There exists a strong positive customer lifetime profitability relationship

- This was supported but only a moderate linear association was found. Approximately $40 \%$ of customers fell out of the expected quadrants between tenure and profitability.

2. Profits increase over time

- Reinartz and Kumar (2000) asserted that both long and short-term customers form the core of company profits hence the relationship of time and retention to profitability was not supported. As such, the proposition was not supported.

3. The costs of service to long-life customers are less

- This proposition was not supported as they found the mailing cost per dollar sales was not statistically different between short and long-term customers. Notable was that this related to only one dimension of the cost components - mailing. 
4. Long-life customers pay higher prices

- The research finding did not support this proposition. In reality the research found the opposite situation. Short tenure customers paid on average a price of between $8 \%$ and $10 \%$ higher on a single product than long tenure customers.

\subsection{Customer retention calculation}

In their article Reinartz and Kumar (2003) discussed the issue of calculation and then presented a conceptual discussion on the calculation input variables. They suggested the modelling may be improved through using Schmittlein and Peterson's (1994) customer lifetime duration measurement. Through adding Mulhern's (1999) variable ' $\mathrm{P}$ (active)' to represent the probability a customer is still active in purchasing a companies product. In short - do you still have a relationship with that customer or is their 'lifetime' with that firm expired. Reinartz and Kumar (2003) put forward this as a suitable treatment to the calculation for customers in non-contractual relationship settings. This is an important contribution to enhance the calculation as it introduces consideration that, in a non-contractual setting, customers are not as reliable as those contracted. Hence this vulnerability or reduced certainty needs a treatment.

A notable addition to the body of literature on Customer Lifetime Value (CLV) calculation was Pfeifer and Carraway's (2000) article. Pfeifer and Carraway's (2000) contribution set out to address their perceived limitation in the model by Berger and Nasr (1998) where no consideration was given to probability that a customer would 
repeat purchase in the future. This under the Berger and Nasr (1998) model was assumed as given. This was suggested by Pfeifer and Carraway (2000) as limiting the models practical application as it did not consider broader factors that can influence a consumer's purchase decision making. The addition to the model added a calculation of probability that a customer will repeat purchase behaviour in a future period. Pfeifer and Carraway (2000) applied a general class of mathematical model called the Markov Chain Model (MCM). The model creates probabilities of a customer moving from one decision state to another in a single period. Figure 2 is a graphical representation of a firm's relationship with a customer over a five year period context using the MCM approach. The customer at the fifth period is deemed to have become a 'non-customer' or 'former customer' as depicted with the probability of being a customer in the next period.

Figure 2: Markov Chain Model (MCM) calculation

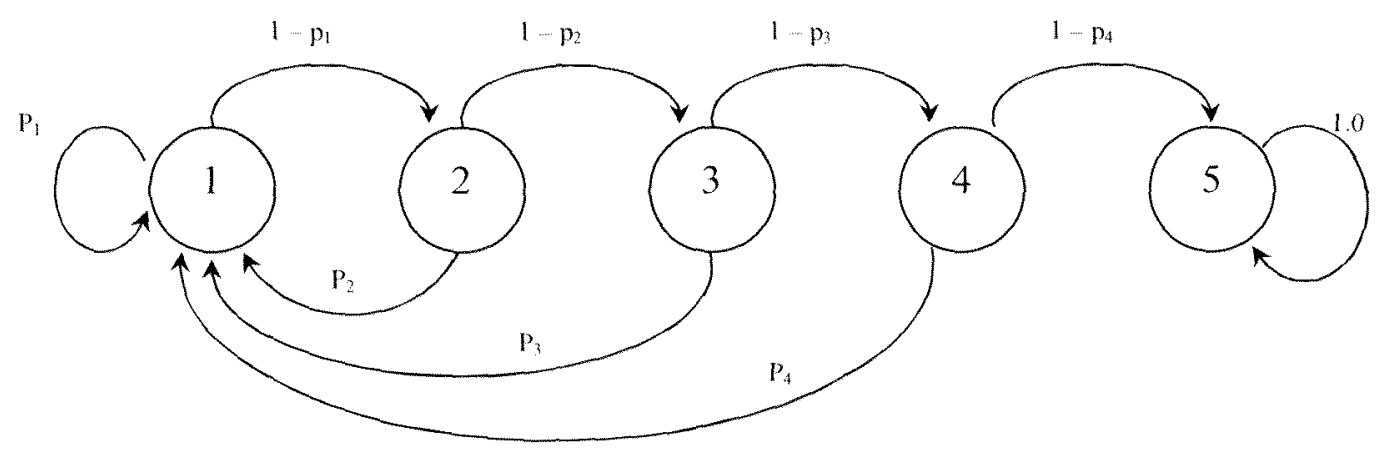

(Pfeifer and Carraway, 2000, p.45)

Pfeifer and Carraway (2000) noted the MCM's flexibility as the key advantage of using this approach over other models to provide more accurate inputs to handling retention, migration and acquisition situations. The model is introduces the element of 
probability thereby explicitly accounting for uncertainty of customer relationships. Pfeifer and Carraway (2000) combined the MCM model with the RFM framework and tested the model with catalogue data from the USA to illustrate the application.

Although the introduction of the probability of future purchase has potential to enhance the CLV model, it has not been subject to broad empirical testing. The addition of MCM may be found to enhance the model.

The MCM model in this paper focused on the application to managerial decisionmaking and not specifically to CLV determination. Further testing is considered necessary to clarify the accuracy and applicability of this MCM approach and value to the context of CLV calculation.

Libai, Narayandas and Humby (2002) in their paper presented a number of limitations to Berger and Nasr's (1998) model when applied in the context of Jackson's (1985) lost-for-good customer scenario - where a customer makes a longer term commitment to an organisation where switching costs are high. Libai et al. (2002) found that the CLV model examined did not assist managers in practical case scenarios and hence limited its adoption. This has implications for high frequency purchase products (such as Fast Moving Consumer Goods - FMCG) where a customer may operate from a repertoire of brands with equal loyalty. This was highlighted in Berger and Nasr's (1998) suggested areas for future enhancement, noting that adding brand loyalty and customer satisfaction variables into the calculation should be explored. 
Jain and Singh (2002) presented a good overview of literature as observed at that point in CLV model and theory development, summarising the state of the CLV model development. The article also outlines a number of limitations to Berger and Nasr's (1998) approach to CLV calculation such as the amount of cash flow from a customer, timing of cash flow, the type of business model, and the type of data needed. Jain and Singh (2002) also noted the lack of empirical validation observed in the current body of literature on CLV model development. Further extension of the CLV model is suggested by Jain and Singh (2002) to broaden model inputs to include demographic information and product usage variables. They note that not all models available at that time need further development to be applicable to product categories.

Reinartz and Kumar (2003) article continued development building on Reinartz and Kumar (2000) where they incorporated the projection of profitability with the computation of lifetime duration. They compared the RFM framework to the model of customer profitability proposed in Reinartz and Kumar's (2000) model. Whilst the results are not presented, there is the position taken that Reinartz and Kumar's (2000) model delivers superior accuracy in its predictability or variance in measurement of the value than using the RFM approach.

The article by Reinartz and Kumar (2003) also responded to the findings of Reinartz and Kumar (2000) paper by conceptually reviewing antecedents that can influence calculations of lifetime duration. Their paper tested several propositions. 
- The level of spending is positively related to profitable customer lifetime duration.

- The degree of cross department buying is positively related to profitable lifetime duration.

- Lifetime was shown to be shorter when the time between purchases by a customer was inconsistent.

- The proposition that higher company profits had a correlation to greater customer dissatisfaction was not supported. Finding that the relationship to company profitability had more to do with the degree of spending by customers and not satisfaction.

- Loyalty schemes are associated with higher lifetime value.

- Profitable lifetime value is supported in the mailing effort of direct marketing activity.

- Profitable customer lifetime duration is higher for customers living in areas with lower population density.

- Age is not related to profitable lifetime but income is.

Reinartz and Kumar (2003) were successful in outlining a number of conceptual influencing antecedents that should be considered. However, Reinartz and Kumar's (2003) article did not provide support for the weighting factor associated with the antecedents themselves on the calculation. They highlight that "customers are heterogeneous on an important relationship characteristic - lifetime duration" (Reinartz and Kumar, 2003, p.23). Their research found that customer heterogeneity 
was not isolated to the characteristic of tenure. Other CLV Model characteristics such as consumption also varied.

At a conceptual level Reinartz and Kumar (2003) were successful in providing 'reasonable doubt' that Reinartz and Kumar's (2000) findings may not be accurate. This finding supported Berger and Nasr's (1998) original DCF concept.

Rust, Lemon and Zeithaml (2004) presented an excellent overview of the body of literature in this area and distinguished three streams of development in relation to Customer Lifetime Value. They are:

- CLV models (e.g. Berger and Nasr 1998);

- Direct marketing-motivated models of customer equity (e.g. Blattberg and Deighton 1996), and

- Pfeifer and Carraway 2000)

- Longitudinal database marketing models (e.g. Reinartz and Kumar 2000)

Rust et al. (2004) proposed a model that builds on the above streams, not relying on the input of longitudinal data being. The proposed model by Rust et al. (2004) was more general and incorporated all marketing expenditure not purely direct marketing expenses. The model was ambitious, in that it incorporated competition and brand switching elements. The following model was proposed by Rust et al. (2004) for calculation of CLV for the subject customer to a specific brand. 
Figure 3: CLV model by Rust et al. (2004)

$$
C L V_{i j}=\sum_{t=0}^{T i j}\left(1+d_{j}\right)^{-t / f_{i}} V_{i j t} \pi_{i j t} B_{i j t}
$$

(Rust et al., 2004, p. 114)

Where:

i Is the customer

j Is the firm

$\mathrm{f} \quad$ Is the frequency of purchase of customer $i$

1 Is the moment of purchase in time

d Is the discount factor of the firm

$T_{i j} \quad$ The number of purchases customer $i$ is expected to make before firm $j$ 's time horizons

Vijt Customer i's expected purchase volume in a purchase of brand $\mathrm{j}$ in purchase $t$

mijt $\quad$ Expected contribution margin per unit of firm $\mathrm{j}$ from customer $\mathrm{i}$ in purchase $\mathrm{t}$

Bijt The probability that customer $i$ buys brand $j$ in purchase $t$

An important contribution by Rust et al. (2004) to CLV model calculation development is their introduction of competitor offerings and brand switching variables (calculated through application of Markov Chain Modelling) in the formulae proposed by Pfeifer and Carraway (2000). The CLV model proposed is based on the discounted cash flow methodology used by Berger and Nasr (1998) and incorporates a utility formulation of brand and competitor inertia being the introduction of frequency, margin, volume and brand choice probabilities to a customer purchases in this 
calculation. Additionally, the model incorporated a probability of choice creating an input switching matrix at the individual level.

Rust et al. (2004) then applied the new model to calculate the customer equity and ROI analysis, utilising outputs of their refined CLV model. Rust et al. (2004) utilised cross sectional data to establish customer ratings of competitor brands and brand purchase behaviour. Customer ratings of brands is achieved through observation, panel data or using purchase intent as a proxy for profitability in the next intended purchase. This is applied to each purchase for each brand in a repertoire. Once the variables are established they use multinominal logit choice model analysis to establish driver levels.

When comparing the model proposed by Rust et al. (2004) to other CLV models such as Berger and Nasr's (1998), it was found that "the lost-for-good [being Berger and Nasr's (1998) approach] model provides a systematic underestimation of customer equity that, in this case, is an underestimation of 47.3\%" (Rust et al., 2004, p.121).

This is an important finding, yet Rust et al. (2004) did not articulate which model was the more accurate. Of interest in Rust et al.'s (2004) model was it discounts according to individual purchases, and moves away from the risk identified in this research of acting at aggregated levels. Several limitations were outlined in the article including the impacts of cross-selling and competitor reactions, which were acknowledged by Rust et al. (2004) areas of future development. 
An observation of the contribution made by Rust et al. (2004) is the challenge of incorporating individual (transaction or consumer) level variables. Consideration should be given in development of calculation models to the practical application. If consideration is not given then the contribution to the theory is constrained. Rust et al.'s (2004) model suggests application at individual consumer or transaction level. This presents an unlikely scenario due to the practicality of using the model and accessibility to the input information in a way that easily allows for aggregation and decisions to be made at a segment level.

\subsection{CLV and customer equity}

During the development of CLV literature Pfeifer, Haskins and Conroy (2004) addressed the issue of terminology. They identified the need for clarification between the expressions 'customer lifetime value' and 'customer profitability' when referenced and set out to illustrate the key differences.

Effectively Pfeifer, et al. (2004) distinguished customer lifetime value as a calculation that should not include acquisition costs. Where acquisition costs are included they support Blattberg and Deighton's (1996) model that calculates the profitable customer lifetime value where a firm has expenditure prior to receiving revenue from the acquisition.

There is a distinction introduced by Kumar, Ramani and Bohling (2004) where CLV may be applied to the contexts of averages and to individual customer situations. 
Kumar et al.'s (2004) article set out clarifying the difference between averages and individual situations and considerations that should be undertaken for calculating CLV in the two contexts.

Kumar et al. (2004) noted that in the context of measuring CLV for a group of customers at an aggregated level, the approach outlined by Berger and Nasr (1998) was supported. They did not suggest changes were required to the model for that application. They identified a key benefit of aggregating the data for a set of customers was for evaluating at a competitor firm. This allows insight and comparison with competitor firms. Additionally this approach was proposed to assist with merger and acquisition decisions (Gupta and Lehmann, 2003; Gupta, Lehmann and Stuart, 2004).

Kumar et al. (2004) reviewed the context of individual level lifetime value calculations and suggested the application of a probability element (the P(Active) probability) required to reflect the active status of the customer, as outlined in Schmittlein, Morrison, Colombo (1987) and Reinartz and Kumar (2000).

In preferring this approach Kumar et al. (2004) illustrated several managerial applications for their proposed model in areas such as customer selection, management in relation to investment, and resource mix and frequency of communication applications. It is not clear why Kumar et al. (2004) suggest that the two contexts of aggregated and individual level calculation could not be accommodated under one model. They infer that introduction of a probability component, used in the individual 
model, may present improved accuracy however they do not provide empirical support for this assertion.

Kumar et al.'s (2004) article did not specifically contribute to CLV formulae calculation in the context of aggregated or individual level contexts but provides an opinion on preference. The article responded to criticism by Jain and Singh (2002) expressing concern about the limitations of practically applying CLV models. The development of the solution was not limited to customer lifetime valuation alone. The following paper investigates how calculating the lifetime value of a firm's customers can play a role to the in valuation of that firm.

Gupta et al., (2004) paper proposed using the calculation of the customer lifetime value of a firm's customers to estimate the value of a firm. They investigated the discounted cash flow approach and tested calculation of CLV for a firm's customers against the company annual report data. A key finding in their research was to identify that "retention rate has a significantly larger impact on customer and firm value than does discount rate and cost of capital" (Gupta et al., 2004, p. 17). The element of retention rate is examined in their research investigating its role in calculating CLV.

\subsection{A product perspective}

Up until van Triest (2005), the body of literature centred on the CLV calculation applied to segment, aggregated, or individual (i.e. customer or transaction) level contexts. The contribution by van Triest (2005) was to approach the customer lifetime 
value calculation differently, investigating the impact of changing the relationship to that of customer profitability at product level.

Van Triest (2005) introduced a generalised customer profitability model to explore the relationship between customer size and customer profitability. Assuming there is a correlation between size and increased profits, van Triest (2005) sought to identify where a higher profitability margin may come from. Van Triest (2005) introduces a model with several variables relating to organisation size, purchased products (and product margin), sales, exchange efficiency, tenure, and purchase volume. The propositions proposed by Van Triest (2005) related to if larger organisations achieve greater product margins (discounts), have less support demands and higher exchange costs. The findings did not support the examined proposition that large organisations generate greater product marginal profit but did posit that the size effect is relevant to exchange efficiencies.

\subsection{The context of risk}

Ryals and Knox (2005) make a unique contribution to the body of literature by investigating a way of 'risk-adjusting' the calculation of customer lifetime and customer profitability. Ryals and Knox (2005) applied a risk element in profiles specifically to the revenue component where other authors such as Pfeiffer and Carraway (2000) and Levett et al. (1999) applied it to other elements, specifically the customer and their probability to be retained. From the result of that step Ryals and Knox (2005) propose calculating the Economic Value (EV) measurement of a 
customer as a product of combining a forecast of the CLV and the future customer risk relative to revenue.

The approach taken by Ryals and Knox (2005) calculates risk-adjusted revenues, subtracts cost and adjusts to present value using the weighted average cost of capital. It effectively takes an approach to reflecting the risk in the relationship by applying a 'risk adjustment' to the revenue component. The paper argues that the difference between risk adjusted and non risk adjusted is the difference between the CLV calculation and the EV. Ryals and Knox (2005) apply the methodology to insurance data to outline the difference and consequently discussed the marketing management implications to decision making.

More recently, Haenlein, Kaplan and Schoder (2006) built on the collection of work of CLV calculation, maximising marketing resource allocation (Blattberg and Deighton, 1996; Berger and Nasr, 1998; Reinartz, et al., 2005; Rust, et al., 2004; and Venkatesan and Kumar, 2004) and use of Option Theory (Levett et al. 1999) to introduce a new model. Combining the CLV approach proposed by Berger and Nasr (1998), and real option analysis (specifically the abandonment option), Haenlein et al. (2006) ranked customers to guide managerial decision on resource allocation.

Haenlein et al. (2006) presented the proposition that using traditional discounted cash flow based CLV models to direct allocation of scarce marketing resources, as suggested by Blattberg and Deighton (1996), results in flawed and biased outcomes. The article suggests that the use of real option analysis combined with CLV analysis 
can lead to more efficient use of resources. This approach introduces the flexibility for the firm to remove (or explicitly not target) unprofitable customers.

Haenlein et al. (2006) undertook testing of the propositions using longitudinal data from a USA catalogue company over a 12 year period. The research calculated purchase frequency, cost of sale, and marketing activity per customer. With these inputs established future profit contribution is estimated. Calculation of these inputs enabled application to a standard CLV under the scenario that includes real option value and without the addition of that real option element. Haenlein et al. (2006) reported a consistent underestimation of customer value.

The consistency in the error (being underestimation of customer value) identified in Haenlein et al.'s (2006) research provided opportunity to investigate the source of variation. For this purpose they undertook Monte Carlo simulations and identified divergence increases with decreases in future purchase probability and decreased future expenditure.

One of the challenges in the work by Haenlein et al. (2006) was the introduction of a highly complex calculation model that integrated the Option Theory elements into the CLV calculation. An observation is that the complex nature of the model inputs would prove challenging in a practical context. 


\subsection{Literature review - summary}

In summary, the body of literature on CLV calculation has developed with a relatively narrow extension of the core theory and methodology either from Recency, Frequency, and Monetary (RFM) or Discounted Cash Flow (DCF) foundations and the model introduced by Berger and Nasr (1998). The variations in literature are observed to focus on extending the way the calculation of model inputs are made and the use of various inputs in the model. Additionally the aspect of probability of future transactions and customer retention received strong attention.

A weakness identified from this literature review is the lack of application and empirical validation of many of the conceptual proposed models. In selecting the approach to use in this research early articles by Blattberg and Deighton (1996) and Berger and Nasr (1998) presented a clear foundation. The CLV calculation model proposed by Berger and Nasr (1998) was popular in literature and continues to be the basis for future development of model calculation approaches. Contributions in literature following Berger and Nasr's (1998) model, tended to focus on ways to increase the accuracy in predicting model inputs and a future customer state of tenure or propensity to purchase. Additionally, several authors explored how managers may use CLV calculation results to make strategy decisions and apply scarce resources. The model proposed by Berger and Nasr (1998) was a clear choice for testing the reliability and accuracy of the CLV model to predict future states of customer value. This was because the CLV calculation model proposed by Berger and Nasr (1998) was 
the highest cited article in the body of literature on this topic and from observation in this review as well regarded and referenced as a foundation approach.

There were however two key areas of enhancement to the CLV models reviewed in the literature. Several attempts were made to improve the retention rate or customer tenure calculation inputs. The literature discussed the use of methods such as Markov Chain Modelling (MCM) for calculating customer duration and introduced consideration of risk into the equation. The use of option theory was also suggested as an enhancement to the model. Both these proposed additions focus on the probability of future purchase. In both instances there was very little empirical support and in general their contributions were of a conceptual basis. It is surprising given the desire marketing managers and academics have expressed in the literature to develop models to financially account for benefits in marketing decision making. Overall, it was observed that there is a relatively limited amount of empirical testing presented in the literature, with a disproportionate amount of conceptual papers contributing to the model development literature in this area.

The application of the CLV models were incorporated to illustrate decision making about customers, segments, products and discuss inputs/variables that may influence the outcomes and impacts on managerial decision making. The momentum of new contributions has continued under the assumption the foundation model(s) were valid and accurate. These have tended to extend further Blattberg and Deighton's (1996) discussion on resource allocation decision making. 
The primary contribution of this research is empirical testing of the established Berger and Nasr's (1988) CLV calculation model. This research investigates the predictive accuracy of the model at both aggregated and segment levels and assesses the fit between forecast and accurate calculations. In addition, the study examines the input variables to the model and the influence they have on the CLV model calculation outcome. 


\section{Research question}

This study empirically examines Berger and Nasr's (1998) Customer Lifetime Value (CLV) calculation model, identified as a foundation CLV calculation from review of literature. The purpose of this research is to contribute to academic and managerial confidence that suitable rigor has been applied to Berger and Nasr's (1998) CLV calculation model. The validation of this model is important as it is the basis from which further theory has developed in the area of CLV calculation.

\subsection{Principal research question}

There are several elements to this research. The primary goal is to test the accuracy and reliability of Berger and Nasr's (1998) model and the sensitivity of the models input variables. Secondly, a number of propositions have been identified from the literature and this research presents opportunity to also empirically examine these. The research question is presented and examined in two parts:

Q1

What is the accuracy of the Berger and Nasr's (1998) CLV calculation model to predict a future state (fit - between the model and actual data based calculations)?

Q2

How sensitive is the Berger and Nasr's (1998) model calculation output to variation with the input variables? 


\subsection{Related propositions for examination}

In review of the literature several relevant propositions to the principal research question were identified for examination. The propositions are detailed below. Propositions 6 and 7 did not come directly from literature. The access to available data in this study has provided opportunity to examine these questions.

P1 Businesses lose $15 \%-20 \%$ of their customer each year.

This proposition arises directly from a claim by Reichheld and Sasser (1990).

P2 Companies lose half their customers every five years.

If proposition 1 is accepted then it follows that this proposition by Reichheld (1996), based on their research of USA corporations, follows and can also be examined. Whilst propositions 1 and 2 are linked, the purpose for treating these propositions (P1 and P2) independently is because they were identified in different papers.

P3 Customers who stay longer increase cash flow over time (cash flow being the incremental revenue received from sale of a product or service).

Reichheld and Sasser (1990), Reichheld and Teal (1996), and Berger and Nasr (1998) observe that customers who stay longer increase cash flow, as sales (revenue) earned by a firm from a customer over the period of time of the relationship, and profits over 
time. This challenges the assertion of Dowling and Uncles (1997) particularly in the context of non-contractual settings. The proposition for examination in this research is limited to cash flow. There are a number of inputs to profitability and all the contributing elements of the profit equation were not available from the data source Rata Energy.

This proposition is similar to the findings of Reinartz and Kumar (2000) who noted two relevant findings. Profits were reported to increase for a customer over time and a correlation was found between tenure and profitability. These findings were not directly examined in this research due to missing input data on customer profitability.

P4 Retention rate is the most critical input variable in the CLV calculation.

This proposition is derived from Gupta et al.'s (2004) research. They found that the retention rate, being the ratio of likelihood a customer will be retained in a following period, had a larger impact on customer and firm value than the discount rate or the cost of capital used in the CLV model tested.

P5 Different customer segments exhibit different patterns of attrition.

This proposition arises directly from Wyner's (1999) paper where they state different customer segments exhibit different patterns of attrition, switching and reactivation. This research is limited to testing part of the overall proposition by Wyner (1999), due to the lack of suitable input information on switching and reactivation from Rata 
Energy. Wyner (1999) noted that this presents considerable risk to the accuracy and role of many subsequent enhancements of the CLV model.

P6 Using input variables (based on actual 2003 input data) derived at segment level will result in more accuracy in the CLV model calculation.

P7 The use of an average retention rate derived from the actual cohort data will lead to highly accurate results.

Propositions 6 and 7 arise from the logic that a greater level of detail will lead to improved accuracy in the CLV calculation. The availability of actual data over the four year period from the Rata Energy provides the unique opportunity to not only calculate actual retention rates, being the ratio of likelihood a customer was retained in a following period, but provide the ability to segment the cohort for further analysis. 


\section{Methodology}

This study identified from literature a suitable 'foundation' customer lifetime value calculation model. In addition, relevant propositions identified in literature will also be examined.

Data of four years from 2003 - 2006 inclusively, is profiled using descriptive statistical analysis to better understand the cohort characteristics. The profiling of the data is done at aggregated and segmented levels. The key research problem is explored following a three step process of calculating CLV for a group of customers over a projected period of 3 years. The next step was to calculate the actual value of that cohort using actual data for the 3 year examined period. Lastly, the two sets of results are compared to identify the level of variance and make related assertions as to the level of accuracy the CLV model provides.

In addition, the sensitivity of the chosen CLV model to change in the input variables is examined to understand the level of influence each variable has on the outcome of the CLV model's calculation. The sensitivity of the input variables was examined to $15 \%$ variation in the calculation result as by industry standards would be perceived as considerable. The sensitivity analysis led to several propositions examined that related to this topic of CLV calculation identified form relevant literature and presented in Section 3.2 . 


\subsection{Research design}

The research whilst using empirical data is exploratory by nature. It uses statistical analysis to determine how closely the actual calculation findings compare to those made by the Berger and Nasr (1998) model and examine sensitivity characteristics of the input variables in the model when in the context of a comparison to actual data. A finding of more than $10 \%$ variance by industry standards would be perceived as considerable and would put in doubt the value of the calculation findings to business decision making.

This research adopts the approach to customers introduced by Jackson (1985) where customers are either part of lost-for-good or always-a-share segments - this approach was used as it represents a common context taken in literature for the application of Berger and Nasr's (1998) CLV model. The specific context for this research was the Lost-for-good segment. This assumes a customer is either totally committed to the vendor or totally lost and committed to another vendor. This means there is no 'switch back' activity as part of this customer group. This approach was adopted to simplify the context of the model evaluation, and enable clear assessment of the model fit and sensitivity around input elements. Berger and Nasr's (1998) model used in this study application to this cohort and based on the lost-for-good scenario. 


\subsection{Data}

The data extract was sourced from a large energy retailer in New Zealand which for the purpose of this research is called Rata Energy. The extract of customer data for the cohort was taken from the core transaction billing system for the use in this research was extracted March 2007. The cohort data extraction was from 1 January 2003 to 31 December 2006.

The cohort data from Rata Energy was provided in a way as to ensure no compromise of Rata Energy's customer privacy by using only two data elements for each customer being:

- Account number (allocated by the Rata Energy billing system)

- Consumption volume in kWh (kilowatt hours) each month for each consumer.

Initially, 'billed amount per month' was provided in the extract but this was rejected due to a number of inconsistencies in the recorded financial data (not present in the energy volume data). Additionally, the risk of utilising the billed amounts included a highly complex approach to applying various network charges and other levies that did not necessarily reflect a consumer's consumption activity. The measurement of $\mathrm{kWh}$ (kilowatt hours) per month was the preferred means of representing customer activity.

The initial data extract contained 28,492 suitable customers. The data did require 'cleaning' to ensure no switch-back consumers or general data anomalies were 
included. A switch-back is a customer who had a gap in consumption data recorded. A customer was also deemed to have defected was taken as someone where there was no longer recorded consumption for that customer in that year or subsequent periods.

Following consultation with Rata Energy, 272 consumers captured in the original extract were removed. In addition to any evidence of switch-back, customers were removed from the extract due to discretions in the data held. It was reported by Rata Energy that these data discrepancies were not usual and most likely a result of billing processing or internal data transfer anomalies. An example is where power meter multiplier errors impact on the billing calculation process. The result of the cleansing process was a final extract reduced from 28,492 to 28,220 customers for the final cohort.

Other input variables such as marketing spend per customer per annum and the discount rate, were sourced directly from Rata Energy. These were attained from either historical records or by interview with key marketing and finance staff.

\subsection{Limitations}

\subsubsection{Marketing spend}

Rata Energy only had recorded marketing expenditure on their residential consumer base available for the year 2006 (this was due to poor record handling and the transition of several internal operating systems over the period data relates). After 
consultation with the management of Rata Energy, it was identified that the marketing expenditure was a relatively consistent amount budgeted from preceding years. Rata Energy provided the spend on marketing per customer for 2006 and endorsed the use of that amount as a proxy for the preceding periods which covers the period of this study.

\subsubsection{Costs}

The cost to serve customers was not provided by Rata Energy. This did not impact on the ability to undertake this examination. However, the absence of accurate cost input data limited the ability of this study to test propositions observed in literature on the profitability of a customer and the potential reduction of cost to serve over the lifetime of a customer.

\subsubsection{Value}

The pricing information is very complex and was not available at a detailed level. A suitable extraction was not possible of the related network charges and other associated charges relating to a consumer account. A contribution per $\mathrm{kWh}$ was identified as $\$ 0.37$ per kWh following consultation with Rata Energy's finance team. This figure was applied to all consumption data to provide a monetary representation of the value of the customer. 


\subsubsection{Customer tenure}

A customer was deemed to not be a Rata Energy customer when their annual consumption was nil. The consumption data for the cohort was calculated and aggregated to an annual figure per customer. Where a customer left during a year they were still counted as a customer and not lost until the following year when they would have a 'nil' consumption recorded. 


\section{$5 \quad$ Results and discussion}

\subsection{Introduction}

In review of the relevant literature on this subject, Berger and Nasr's (1998) CLV calculation model is the selected model to be applied in this research. The context for this research is the residential energy retail sector, specifically 28,220 customers of the company Rata Energy. The analysis as outlined in the methodology has been undertaken and this chapter presents the findings and relevant discussion. The results, observations and discussion are structured into four parts:

1. Cohort description - descriptive statistics of the cohort including a profile of the relevant general market conditions (retention and consumption over time at an aggregated level).

2. General application of Berger and Nasr's (1998) model and appropriate fit (actual vs. modelled) assessment.

3. Examine the input variables used in Berger and Nasr's (1998) model to understand the level of sensitivity and how the model is influenced by change in the input variables (retention, marketing expenditure and discount rate). 
4. Take a closer look within the cohort to provide greater insight into the customers and possible implications on the models effectiveness and research findings. Including further testing of Berger and Nasr's (1998) model using segments identified within the cohort and actual based input variables.

\subsection{Cohort description}

\subsubsection{General market conditions}

The extract of customers used in this cohort was taken from the New Zealand energy sector. The subject company, Rata Energy produces an annual report that captures observations about the electricity sector and the following extract presents a context of the market conditions for this study. "New Zealand's demand for electricity has grown consistently over the last 20 years. Electricity consumption has increased from approximately $27.7 \mathrm{TWh}$ (Terawatt hours) in 1985 to $41.5 \mathrm{TWh}$ in 2005 , an average growth rate of 2.2 percent per annum" (Rata Energy, 2007, p. 6).

The electricity sector in New Zealand has gone through considerable change in the last few years, which resulted in varying levels of switch activity over that period of time. With deregulation in 1999 there was a short period (one-two years) of high switching activity (with up to $30 \%$ customer loss estimated by Rata Energy management) as the companies jostled for market share. The most notable period of unusual activity was in 2001 where lakes were at critically low levels and consumers experienced forced 
black-outs by network companies to manage the scarce electricity resource. This occurred again in 2006 when New Zealand experienced one of its driest winters in 30 years, however the industry took a more proactive approach that year, learning from 2001 and consumer marketing promotions widespread power black-outs were avoided.

From observations by Rata Energy management, the competitive context has settled considerably since deregulation. They were unable to provide specific switching metrics but commented that in the initial years following deregulation in 1999 there was considerable switching activity as consumers came to understand the new market. The new retailers were active in the 'lolly scramble' for customers that resulted from deregulation. In addition there were poor early systems put in place which has led to many down-stream challenges for these relatively young retailers. This soon settled and between the years of 2003 and 2006 (the period of the cohort data extract) the switching activity in the residential consumer market calmed considerably.

\subsubsection{Retention}

A critical dependency on the profitability of a customer over time is the rate at which customers defect or leave the organisation (or where a customer stops buying the product in the context of non-contractual settings). There are a number of managerial decisions that rely on an accurate understanding of the defection rate such as application of scarce resources to acquisition or retention activities. 
Figure 4 presents the number of surviving customers each year for the cohort. It shows a relatively consistent defection of customers and a net loss from 2003 to 2006 of $37 \%$ (10,322 customers). This represents an average annual defection rate of $14 \%$. This is a large number of customers lost - particularly if it is found that these lost customers represent lucrative lifetime customers.

Figure 4: Number of surviving customers per annum

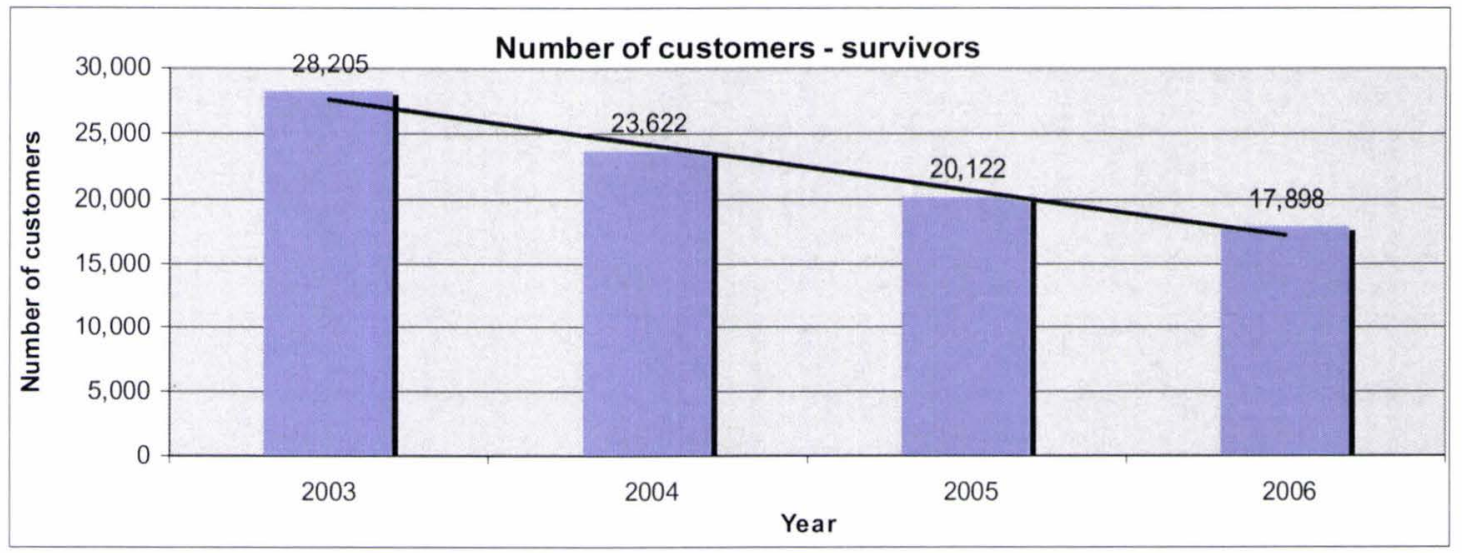

An average defect rate of $14 \%$ as outlined in Table 1 presents an interesting finding when considering Reichheld and Sasser's (1990) somewhat bold and sensational statement that "It is common for a business to lose $15 \%$ to $20 \%$ of its customers each year" (Reichheld and Sasser, 1990, p. 108). Whilst the finding was an average of 14\% this result provides some support for their proposition. 
Table 1: Customer attrition by year

\begin{tabular}{|l|r|r|r|}
\hline & $\mathbf{2 0 0 4}$ & $\mathbf{2 0 0 5}$ & $\mathbf{2 0 0 6}$ \\
\hline Number of lost customers (non-cumulating) & 4583 & 3485 & 2239 \\
\hline \% of original customers lost (non-cumulating) & $16 \%$ & $15 \%$ & $11 \%$ \\
\hline Average of lost customers per annum & & $14 \%$ \\
\hline
\end{tabular}

Table 1 details a relatively consistent reduction in the total cohort - being the level of defection each year of $16 \%, 15 \%$ and $11 \%$ respectively. The rate of decline raises the important question of when the rate is likely to plateau and at what level that is likely to be.

Reichheld and Sasser's (1990) article did not acknowledge the context for managerial decision-making where risk is present in relying on calculations that use aggregated input variables. This was raised by Wyner (1999) when observing the variance that can exist in segments of a customer base. The risk of this is discussed in more detail in Section 5.7 Segment analysis, and investigates variance found in the cohort at a segment level.

The retention of $86 \%$, in the base year, is used for this analysis based on the findings in Table 1. 
Table 2: Cumulative customer attrition

\begin{tabular}{|l|r|r|r|}
\hline & $\mathbf{2 0 0 4}$ & $\mathbf{2 0 0 5}$ & $\mathbf{2 0 0 6}$ \\
\hline Number of lost customers (cumulating) & 4,598 & 8,098 & 10,322 \\
\hline$\%$ of customers lost (cumulating) & $16 \%$ & $29 \%$ & $37 \%$ \\
\hline
\end{tabular}

This research findings presented in Table 2 allow for examining the sensationalised finding of Reichheld (1996) that "On average, the CEO's of U.S. corporations lose half their customers every five years" (Reichheld, 1996, p.56). As the data is only available for the first three years, extrapolation of the data is required to comment on the proposition by Reichheld (1996).

One approach for calculation is to use the average defection rate of $14 \%$ p.a., if this rate was simply extrapolated over a five year period then $14 \% * 5=70 \%$. This represents a higher rate than suggested in the proposition.

Another approach is to use the average \% change between the defection rates. This is calculated as $17 \%$. The $17 \%$ average change in the reduction rate is based on calculating the average $\%$ reduction over the 3 years (with $6 \%$ reduction between years 1 and 2 and a 27\% reduction between years 2 and 3 defection rates). Applying this average reduction of $17 \%$ to the years 4 and 5 , the 5 year defection rates are:

- $16 \%$ - actual year 1

- $15 \%$ - actual year 2

- $11 \%$ - actual year 3 
- $9 \%$ - calculated year $4(9.13 \%)$

- $8 \%$ - calculated year $5(7.47 \%)$

The sum of these defections is a $59 \%$ defection of customers from the original cohort in 5 years. This result supports the proposition based on the variability of the unknown years 4 and 5 . Another recommendation would be to change the proposition statement to include "at least $50 \%$ " to the context of lost customers.

The context of customer switch backs (i.e. a previous customer who left returns as a customer to the organisation) does not seem to be addressed in Reichheld's (1996) proposition. It is not clear if Reichheld (1996) gave consideration to the issue of customers returning during the five year period as this would influence the level of actual 'lost' customers.

\subsubsection{Consumption}

Table 3 illustrates the level of annual consumption activity for the customer cohort. There was a decline in consumption from 2003 to 2004 of $-13 \%$, strong growth in 2005 of $40 \%$, followed by growth in 2006 of $11 \%$. There is an average growth of $21 \%$ for the cohort over the examined period which is considerably more than reported at average industry level with growth of $2.2 \%$ p.a. reported over that period however following consultation with Rata Energy this was put down to the industry level being heavily influenced by significant volume commercial consumers. Table 3 combines 
customer and consumption together illustrating the strong growth in average consumption for survivors.

Table 3: Cohort profile (total customer base)

\begin{tabular}{|l|r|r|r|r|}
\hline & $\mathbf{2 0 0 3}$ & $\mathbf{2 0 0 4}$ & $\mathbf{2 0 0 5}$ & $\mathbf{2 0 0 6}$ \\
\hline Number of survivors each year & 28,205 & 23,622 & 20,122 & 17,898 \\
\hline Total consumption (kWh) & $246,034,288$ & $213,192,263$ & $298,364,470$ & $331,145,770$ \\
\hline \% change in consumption p.a. & & $-13 \%$ & $40 \%$ & $11 \%$ \\
\hline Ave volume p.a. (survivors) & 8,723 & 9,025 & 14,828 & 18,502 \\
\hline Ave value p.a. (survivors) & $\$ 323$ & $\$ 334$ & $\$ 549$ & $\$ 685$ \\
\hline
\end{tabular}

These results present strong support for the relationship marketing paradigm being the tenet that investing in long-term relationships results in benefits to the organisation. Whilst the segments reduce in numbers they increase in total and average per customer consumption.

Whilst not directly relating to the purpose for this research it was appropriate to provide some background to the high consumption activity observed. As such, Rata Energy management were questioned about possible explanations for the variation in the reported consumption and why this was so much higher than growth activity at industry level. Several observations stated as opinions were suggested by Rata Energy management in relation to the difference between industry and cohort consumption growth. At industry level, the proportion of corporate consumers dominates the rate of growth whereas the cohort is an extraction from the residential segment only. This 
does present the risk that a level of selection bias in this research may influence the findings of the analysis.

An additional observation made by Rata Energy management was the change in the residential standard of living in recent times. The introduction of new energy consuming devices in the home such as the shift from fires to heating appliances, 24 hour security systems, or additional appliances could have contributed to increased consumption in the residential space.

The relationship of reported financial value and consumption in this research has been assumed to be direct. The tariffs applied by Rata Energy in usual billing processes were made up of a number of elements. Records were not available on the make up and proportions. For the purpose of this research it is not possible to use actual tariff charges and effectively differentiate the marginal contribution component. As such, contribution is represented by multiplying the consumption volume by a 'per kWh' net contribution amount recommended by the finance team of Rata Energy to generally represent a variable margin figure per $\mathrm{kWh}$.

With an understanding of the retention and consumption profile this research now turns to examining Berger and Nasr's (1998) CLV model for predictive accuracy. 


\subsection{Application of the CLV model - appropriate fit}

The Berger and Nasr's (1998) CLV model used in this study is founded on the accepted method of discounting future cash flows. The model requires a small number of relatively accessible input variables in retention rate, marketing expenditure and discount rate.

It has been observed in this paper that literature on the CLV calculation model has been predominantly conceptual, with little empirical testing. This paper addresses this weakness which is to contribute empirically to the knowledge of how accurate and reliable Berger and Nasr's (1998) CLV calculation is by comparing the model with actual data. The first step in the model application is identification of the input variables.

\subsubsection{Input variables}

The appeal of Berger and Nasr's (1998) CLV calculation model is the relatively accessible input variables, as it does not use subjective variables. This research examines the sensitivity of each of these variables to understand how each potentially impact on the calculation result. 
1. Marketing costs

The approach was to calculate annualised marketing spend per customer by dividing the variable marketing spend for that cohort and dividing by the number of customers in the cohort.

2. Discount rate

This is the company discount rate used in discounting marketing investments (obtained from Rata Energy's finance team).

3. Retention - base year

This variable is a set number used from calculating the retention of customers in period ending 2004. This presents an aspect of risk for the predictive accuracy of the model due to using the retention rate for each subsequent year that was established from the first year. Many authors such as Levett et al. (1999) proposing Option Theory and Pfeifer and Carraway's (2000) application of Markov Chain Modelling were contributions designed to introduce a probability element in the selection of the retention variable. The calculation of retention for use in this analysis is taken at 2004 and is $84 \%$. This is the retention rate variable used for this analysis as prescribed in the Berger and Nasr (1998) approach. 


\subsection{Model calculation compared to actual state}

The testing of the model against actual data for the cohort customers was undertaken in three steps:

1. Calculation of the actual value of the cohort based on consumption data over the examined period using the Net Present Value (NPV) approach.

2. Calculation of CLV for the cohort using Berger and Nasr's (1998) model. This uses the retention rate of the base year (as that would normally be what organisations have on hand - 'this years' retention rate, marketing spend per customer and discount rate). The formulae proposed by Berger and Nasr (1998) presented in Figure 1 calculates gross contribution, then the amount of cost in marketing and subtracts the marketing value from the gross contribution value to reveal the CLV. The discount rate is applied during the two respective calculation steps.

3. Lastly, we compare the findings and calculate the size of variance from actual, average difference and average absolute error. 
The results of the calculations are presented as follows.

Table 4: Model vs. actual comparison

\begin{tabular}{|l|l|l|l|l|l|}
\hline $\begin{array}{l}\text { Actual retention } \\
\text { rate } 2004\end{array}$ & Actual & Model & $\begin{array}{l}\text { \% variance } \\
\text { from actual }\end{array}$ & $\begin{array}{l}\text { Av. } \\
\text { difference }\end{array}$ & $\begin{array}{l}\text { Av. absolute } \\
\text { error }\end{array}$ \\
\hline $84 \%$ & $\$ 1,150$ & $\$ 922$ & $-20 \%$ & $\$ 228$ & $\$ 507$ \\
\hline
\end{tabular}

The result of these calculations shows a sizable variance of $20 \%$ between the actual and model calculations. This leads to the conclusion that Berger and Nasr's (1998) model has not provided accurate reliable measurement of the value of this cohort at the end of 2006. Examining the input variables further may aid in identification of the model calculation variance. This sensitivity analysis is presented in Section 5.5 Sensitivity.

The variance of $20 \%$ observed has a number of potential attributing factors. A key consideration is that the model relies on a constant retention rate variable applied to each year. This differs from the actual occurrence where the retention identified was found to reduce over time and not at a constant rate. This research investigates in Section 5.7 Segment analysis, customers at a segment level and highlights sizable variation between each segment when compared to the aggregated cohort. As such, the use of a constant retention rate and the level of customer heterogeneity present a potential limitation to accurate calculation of the future customer lifetime value. 


\subsection{Sensitivity}

A clever arrangement of bad eggs will never make a good omelette.

C S Lewis (1898 - 1963)

Have we got the right inputs to ensure the CLV model calculation is accurate?

In addition to testing the accuracy of Berger and Nasr's (1998) CLV calculation model, this research examines the input variables relationship to the calculation result. Specifically the sensitivity the model has for each of the individual input variables retention rate, marketing spend and discount rate.

The approach to testing the sensitivity of the model was to identify the level of change in the input variable required to achieve a $15 \%$ change (increase or decrease) in the result of the model calculation. A model calculation result of 15\% was selected as results $15 \%$ above or below would be sufficient to show the influence each variable has on the result. The calculation process used the other input variables consistently in order to isolate the impact of changing only one variable.

The results are presented in three sections (retention, marketing spend and discount rate). Each section will discuss the results of changing that specific variable and the impact on the model outcome. 


\subsubsection{Retention}

Analysis of the retention variable is presented below in Table 5 illustrating the level of variation needed to affect a $15 \%$ change in the model's calculation output.

Table 5: Sensitivity analysis on the retention ( $r$ tn) input variable

\begin{tabular}{|l|r|r|r|r|}
\hline & $\begin{array}{r}\text { Variance in } \\
\text { model \$ result }\end{array}$ & $\begin{array}{r}\text { Required } \\
\text { calculation }\end{array}$ & $\begin{array}{r}\text { Required \% } \\
\text { change in the } \\
\text { model input } \\
\text { variable }\end{array}$ & $91 \%$ \\
\hline $\begin{array}{r}\text { rtn } \\
\text { (retention) }\end{array}$ & $+15 \%$ & $\$ 1,060$ & $84 \%$ & $+9 \%$ \\
\hline & $0 \%$ & $\$ 922$ & $75 \%$ & $-10 \%$ \\
\hline
\end{tabular}

To achieve the target $15 \%$ difference in the model's calculation outcome, the table shows the retention variable would need to be adjusted by $9 \%$ positively or $10 \%$ negatively to get the respective shift in outcome.

There are important implications to managers understanding the level of sensitivity that the retention variable presents in their modelling. It is important as it may influence the way they view the outcomes of the calculation. This analysis provides insight into how Reichheld and Sasser (1990) made the proposition that "Companies can boost profits by almost $100 \%$ by retaining $5 \%$ more of their customers" (Reichheld and Sasser, 1990, p. 105). 
Sharp (2005) presented a paper that should be considered when discussing this finding. It outlined the lack of an empirical foundation to the claim and revealed a number of "wonderfully unrealistic assumptions" (Sharp, 2005, p.2). The assumption by Reichheld and Sasser's (1990) is made about company profitability but uses a basis of customer profitability. Sharp (2005) made other observations that the proposition lacks consideration of costs (such as customer acquisition) in the process. A practical observation was that Reichheld and Sasser's (1990) seem to ignore the ease at which organisations could successfully halve defection rates.

The headline statement that $100 \%$ increase in profits from $5 \%$ customer defect reduction is also challenged by $\mathrm{M}$. Wright, personal communication, March, (2004), in a review of the data presented by Reichheld and Sasser (1990). Where, Wright (2004) observes considerable variation in actual findings from Reichheld and Sasser (1990). To illustrate, M. Wright's, personal communication, March, (2004), reported that the increase in profit from a $5 \%$ customer defect reduction for several industries being far from $100 \%$ such as credit insurance at only $25 \%$. The actual average increase in profit was found to be closer to $48 \%$ than the sensationalised headline by Reichheld and Sasser (1990).

The CLV calculation model's sensitivity to variation in the retention rate over time or between customers presents a risk that decisions such as resource allocation may be made with poor input information. This is in addition to the observed weakness of application of the constant retention rate in this model. Further investigation has been undertaken to understand the subject cohort and possible segments that existed within 
it. The result of this analysis highlights an additional area of concern when using an aggregated retention rate being the considerable variability found between segments within the base.

\subsubsection{Marketing spend}

Table 6: Sensitivity analysis on the marketing (M) input variable

\begin{tabular}{|l|r|r|r|r|}
\hline & $\begin{array}{r}\text { Variance in } \\
\text { model \$ result }\end{array}$ & $\begin{array}{r}\text { Model } \\
\text { value } \\
\text { calculation }\end{array}$ & $\begin{array}{r}\text { Required } \\
\text { model input } \\
\text { variable }\end{array}$ & $\begin{array}{r}\text { Required \% } \\
\text { change in the } \\
\text { input variable }\end{array}$ \\
\hline \begin{tabular}{l} 
(marketing) \\
\hline
\end{tabular} & $+10 \%$ & $\$ 1,024$ & $\$ 0.00$ & $-100 \%$ \\
\hline $0 \%$ & $\$ 922$ & $\$ 26.00$ & $+135 \%$ \\
\hline
\end{tabular}

This analysis examines the sensitivity of the model outcome to change in the marketing variable. The analysis adjusted the marketing variable to the point where $15 \%$ difference in the calculation result was achieved.

The findings show the relative insensitivity of the model change in to the marketing variable. The CLV result was re calculated in each instance reducing the marketing input variable, to the point where the marketing input variable was reduced to $\$ 0$. This resulted in an increase in the CLV of only $10 \%$.

Table 6 illustrates that in order to establish a CLV value 15\% lower than the base calculation then marketing expenditure per customer would need to increase by $135 \%$. 
It is concluded that marketing expenditure in the calculation has a modest impact on the CLV calculation model.

Consideration has not been given to the impact of how marketing expenditure is applied. It is possible marketing efficiency plays a potential role on the level retention based activities that focus on elements such as reducing cognitive dissonance, or introducing loyalty recognition programmes. This would potentially have a positive affect on the level of defects that would take place. The work by Blattberg and Deighton (1996) posit that an accurate understanding of CLV modelling and relevant input variables will allow for accurate decision making for business.

The context of the competitive environment and the characteristics of the financial relationship also have roles in the level of retention and decisions on marketing expenditure investment. In relation to competitive threats organisations may proportionally increase marketing spend relative to the competitive environment to ensure their retention rate stays at an acceptable level - thereby nullifying the impact of the marketing spend on the retention. This would have to assume that organisations make consistent decisions around investment based on competitor threat, or activity in the market.

\subsubsection{Discount rate}

The following table provides analysis on the variation needed in the discount rate to affect a targeted $15 \%$ change in the CLV model calculation outputs. 
Table 7: Sensitivity analysis on the discount rate (d) input variable

\begin{tabular}{|l|r|r|r|r|}
\hline & $\begin{array}{r}\text { Variance in } \\
\text { model \$ result }\end{array}$ & $\begin{array}{r}\text { Model value } \\
\text { calculation }\end{array}$ & $\begin{array}{r}\text { Required } \\
\text { model input } \\
\text { variable }\end{array}$ & $\begin{array}{r}\text { Required \% } \\
\text { change in the } \\
\text { input variable }\end{array}$ \\
\hline d & $+13 \%$ & $\$ 1,055$ & $0 \%$ & $-100 \%$ \\
\hline & $0 \%$ & $\$ 922$ & $8.67 \%$ & \\
\hline & $-15 \%$ & $\$ 783$ & $21.55 \%$ & $+149 \%$ \\
\hline
\end{tabular}

The findings of this analysis show low sensitivity of the CLV model to change in the discount rate. Table 7 illustrates that similar to the findings of the marketing expenditure input variable the discount rate was increased to $22 \%$ in order to realise a drop in customer value of $-15 \%$. In reducing the discount rate it was calculated that even at $0 \%$ there was only a $13 \%$ increase in the value calculated using the model.

\subsection{Summary - model calculation}

\subsubsection{Accuracy of findings}

The analysis on how closely the model calculation compares to calculation using actual data does not support confidence in Berger and Nasr's (1998) model, as reported in Table 4, the variance between actual and modelled calculation was $20 \%$. This indicates a need to investigate further if elements such as the use of aggregated data or the use of constant retention rate have implications on the accuracy the model. 


\subsubsection{Sensitivity - retention rate}

Analysis of the input variables supports Gupta et al.'s (2004) finding that the retention input variable has the most impact on the customer value calculation. This finding supports the proposition (P4) that the calculation of the lifetime value of a customer has a modest sensitivity to retention, and reveals retention is the most influential of the three input variables examined. The model did not accommodate effectively the level of customer heterogeneity in establishment and subsequent yearly treatment of the retention rate.

\subsubsection{Sensitivity - marketing spend and discount rate}

The marketing spend and discount rates will be discussed jointly as the results were found to be relatively similar in relation to the impact on the model. Both input variables had very low influence on the model calculation outcome. The level of change needed to achieve a 15\% variance in the CLV model's calculation result (either positively or negatively) would require such a considerable change in investment that this presents a highly impractical and unrealistic context in business.

The findings of this research leave a number of questions as to the driver for the $20 \%$ variance found in fit between the model calculation and actual data. Additionally, the sensitivity of the model to change in the retention rate variable raises the question; if reducing the size of the cohort would improve the accuracy of the input variables estimated and improve the predictive accuracy of the model. The next stage of this 
study investigates the profiles of identified segments in the cohort and re-applies the model using the smaller customer groups with revised input variables. The aim is to understand if this will have the impact of improving the 'fit' of the model to actual data.

\subsection{Segment analysis}

The headline of a newspaper doesn't always tell the true story...

Having conducted descriptive analysis on the total cohort of 28,205 customers, the investigation turns to understand if there are dynamics within cohort that may impact on the models predictive accuracy. The study profiles segments based on consumption profiles within the cohort. This aims to highlight any weakness in accounting for customer heterogeneity.

The profile analysis of any segments within the cohort provides opportunity to examine the claim by Wyner (1999) that customers exhibit different patterns of attrition. It also enables re-testing of Berger and Nasr's (1998) model using segment level inputs and report if this application produces greater accuracy in the result relative to actual data.

Customers within the cohort were grouped according to their consumption profile. This facilitates examination of the proposition outlined by Wyner (1999) regarding the different behaviours that exist at more detailed levels. The cohort was divided into 
three groups reflecting heavy, medium and light levels of consumption. The three tiers are based on Rata Energy's volume segmentation system.

- Heavy $\quad 10+$ MWh p.a. consumption (8,951 customers 2003)

- Medium $\quad 7+-10$ MWh p.a. consumption (6,939 customers 2003)

- Light $\quad 0-7$ MWh p.a. consumption (12,330 customers 2003)

\subsubsection{Lost customers}

Analysis undertaken in the earlier phase identified a retention rate of $84 \%$ in 2004 , which represents a defection rate of $16 \%$. It was this rate that was applied to the CLV modelling. Under the proposed theoretical approach proposed by Blattberg and Deighton (1996) and Berger and Nasr (1998) organisations may use this aggregated defection rate in managerial decision making.

This research presents a different story. Table 8 presents the findings for the cohort and three segments. The research showed large differences between the total cohort and individual segment levels in 2004 of $5 \%$ (Heavy), 7\% (Medium), and 30\% (Light). This presents a dilemma of what retention rate would be accurate to use for modelling and predicting the value of customers in the future. 
Table 8: Percentage of customers lost each year

\begin{tabular}{|l|r|r|r|r|}
\hline & $\mathbf{2 0 0 4}$ & $\mathbf{2 0 0 5}$ & $\mathbf{2 0 0 6}$ & Average \\
\hline Total cohort & $16 \%$ & $15 \%$ & $11 \%$ & $14 \%$ \\
\hline Heavy & & & & \\
\hline Medium & $5 \%$ & $13 \%$ & $10 \%$ & $9 \%$ \\
\hline Light & $7 \%$ & $15 \%$ & $11 \%$ & $11 \%$ \\
\hline
\end{tabular}

The results presented in Table 8 question what the result of the fit analysis will be using segment specific inputs. Additionally, it may lead to flawed investment decisions when considering use of resources on retention activities. The $16 \%$ defection rate at cohort level would disguise the urgency or scale of investment in marketing activity for retention initiatives needed for the light segment and potentially lead to over-spend on the heavy and medium groups.

\subsubsection{Consumption profile}

Further analysis was undertaken to profile the behaviours of the customers in each segment and show change in segment membership within the cohort over time. Figure 5 illustrates the actual number of customers per segment and the reduction that occurs in light and medium segments and the increase in the heavy segment. 
Figure 5: Number of customers per annum in each segment (2004 classification)

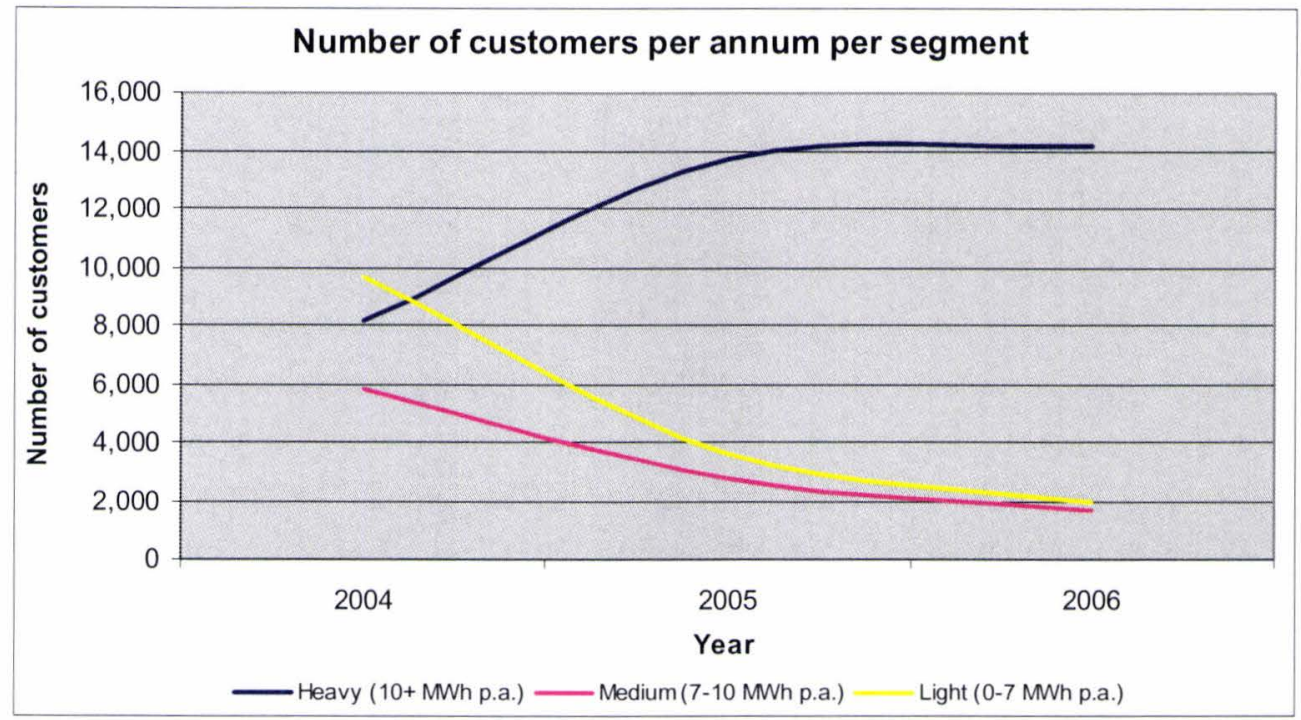

The results shown support the proposition derived from Reichheld and Sasser (1990) that customers who stay longer increase consumption and hence revenue in the form of cash flow (for the purpose of this research regarded as proposition P3). There are some customers who did remain in their respective categories, and a very small number of customers who moved from heavy to medium or light segments. These were interpreted as outliners and the examined proposition P3 is supported.

Figure 6 below is a holistic view of the make-up and change of the cohort over time. The graph illustrates lost and surviving customers by showing the make up of the total customer group over the examined period between 2003 and 2006 with the make-up of years 2004, 2005 and 2006 illustrated based as 2003 being the base year. 
Figure 6: Proportional make up of customer numbers by segment

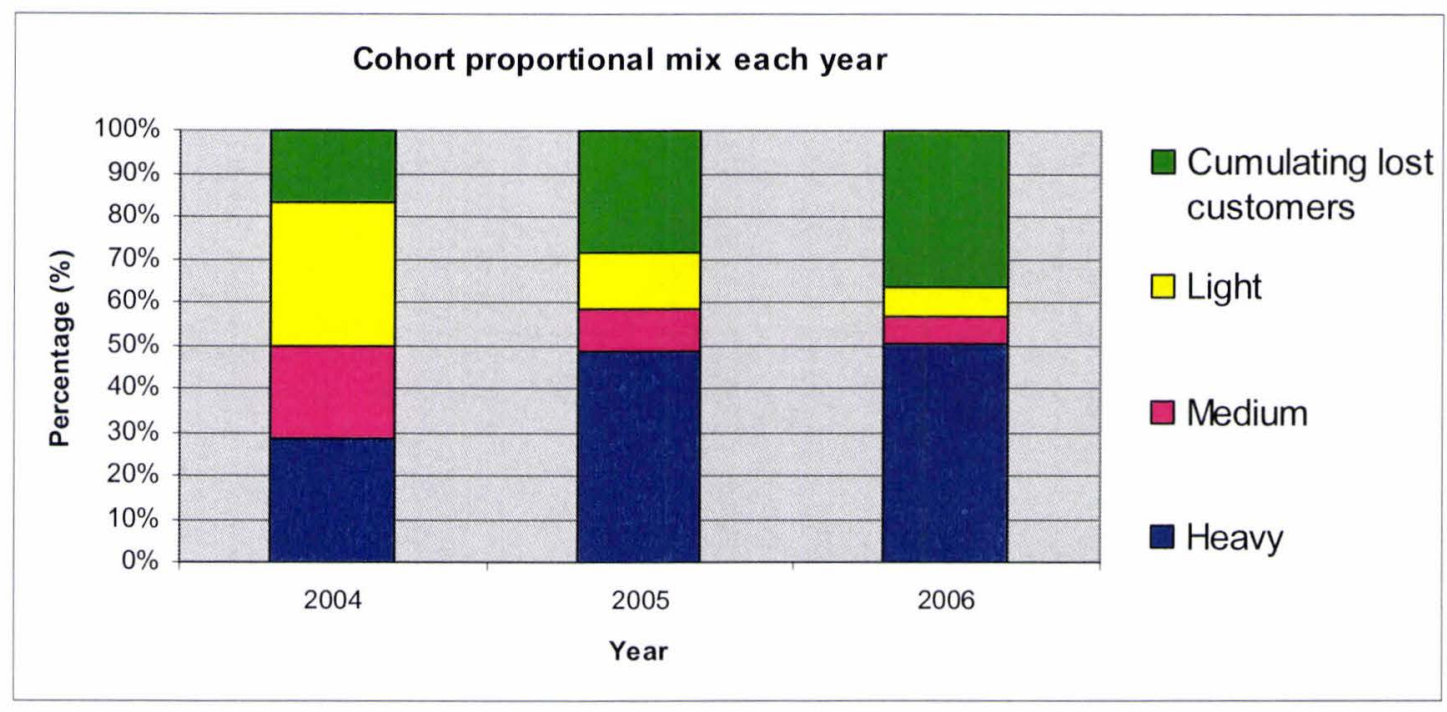

The graph above illustrates the declining number of customers in segments medium (pink) and light (yellow) and it can be observe the growth of the number of customers who fell into the heavy segment (blue) for 2005 and 2006.

\subsubsection{Consumption per customer}

Another way to view these customers is to understand how the make up of the respective segments changes over time if reclassified based on consumption each year. Figure 7 below presents the average consumption per customer and illustrates the relatively steady growth in each segment in average consumption for the customers in each segment each year. 
Figure 7: Average consumption per customer per annum (rolling reclassification)

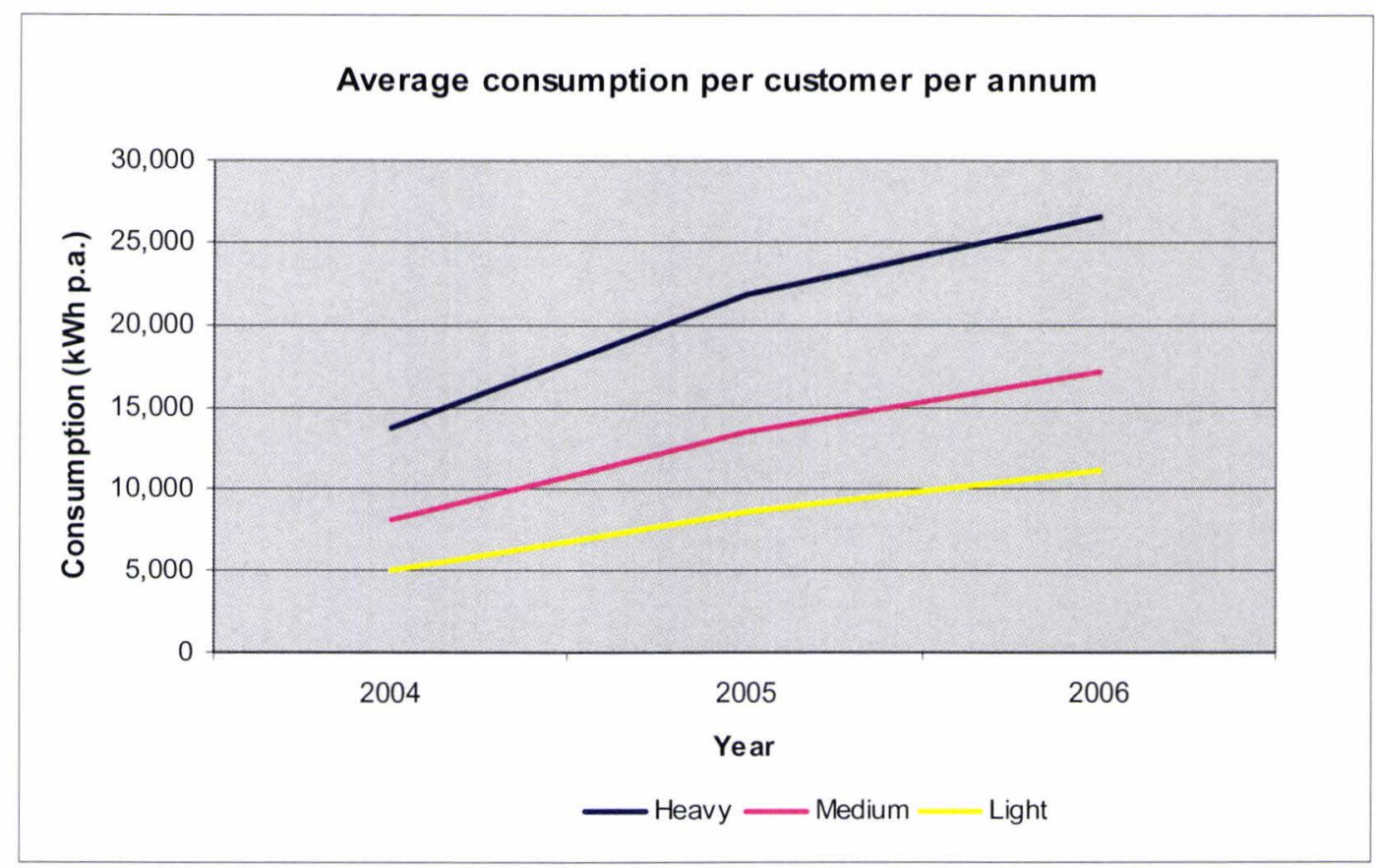

The relationship of consumption to tenure is one of the interesting findings of this research and supports the proposition derived from Reichheld and Sasser (1990) that customers who stay longer increase cash flow. Detailed information regarding customer volume consumption activity is provided below.

The findings challenge Reinartz and Kumar's (2000) paper where they suggest there is no correlation between tenure and profitability. Reinartz and Kumar's (2000) focus was solely on non-contracted settings and as such the context for the study is limited the contractual based customer relationship. 
Table 9: Total cohort and segment profile

\begin{tabular}{|c|c|c|c|c|}
\hline & 2003 & 2004 & 2005 & 2006 \\
\hline \multicolumn{5}{|c|}{ Number of customers } \\
\hline Heavy & 8,951 & 8,136 & 13,715 & 14,197 \\
\hline Medium & 6,939 & 5,848 & 2,794 & 1,706 \\
\hline Light & 12,330 & 9,638 & 3,613 & 1,995 \\
\hline \multicolumn{5}{|c|}{ Total consumption volume by segment ( $k$ Wh p.a.) } \\
\hline Heavy & $136,279,048$ & $117,159,661$ & $161,916,729$ & $177,088,650$ \\
\hline Medium & $58,393,395$ & $52,864,607$ & $74,463,825$ & $83,713,247$ \\
\hline Light & $51,361,845$ & $43,167,995$ & $61,983,916$ & $70,343,873$ \\
\hline \multicolumn{5}{|c|}{ Average volume by customer (kWh p.a.) } \\
\hline Heavy & 15,225 & 13,706 & 21,890 & 26,574 \\
\hline Medium & 8,415 & 8,154 & 13,497 & 17,102 \\
\hline Light & 4,171 & 5,025 & 8,599 & 11,097 \\
\hline
\end{tabular}

\subsubsection{Comparison - ad hoc segmentation}

Organisations have traditionally segmented the customer bases on an ad hoc criteria basis and little understanding is undertaken at the customer level to appreciate change in the customer relationship with a firm. The following illustrates the profile of customers in 2003 by segment and then again in 2006 to compare how the segments are made up. Figures 8 and 9 illustrate the considerable shift in customer numbers for each segment as a proportion of the total customers in that year. 
This section highlights the risk to informed managerial decision making many practitioners face when not tracking individual customer consumption activity and undertaking segmentation in an ad hoc manner i.e. the profitability of a segment at a point in time in contrast to understanding the actual relationship with a customer over time.

Figure 8: Comparing customer number by segment in 2003 and 2006

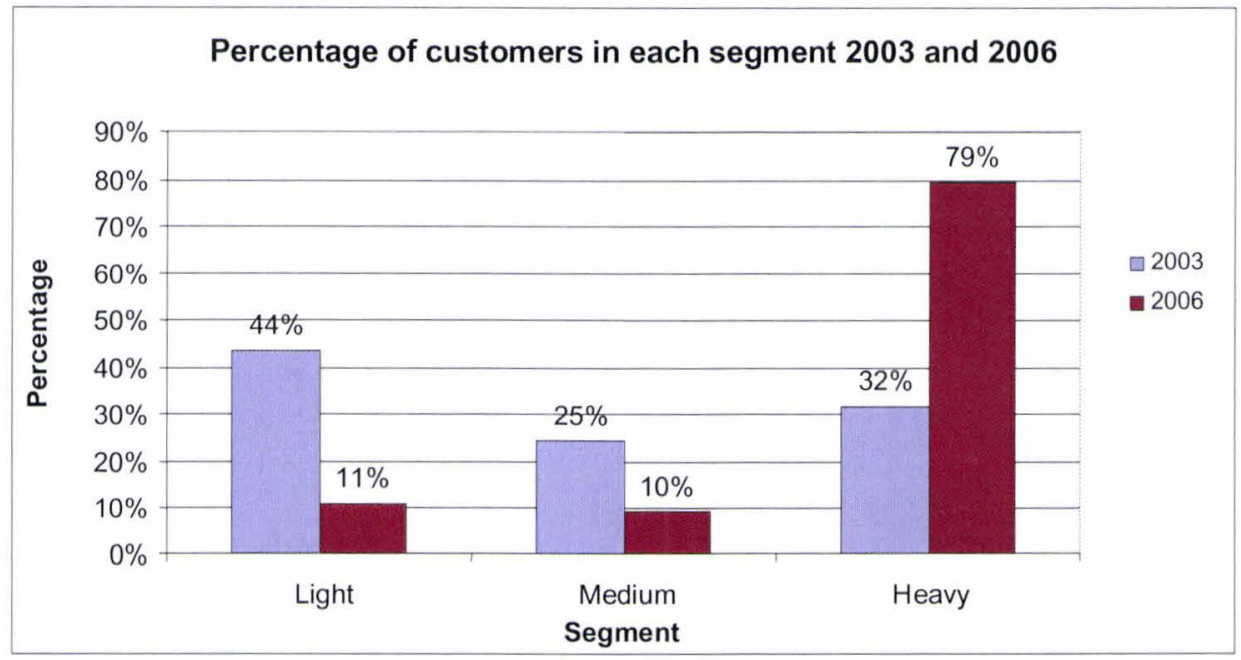

Figure 9: Comparing average consumption by segment in 2003 and 2006

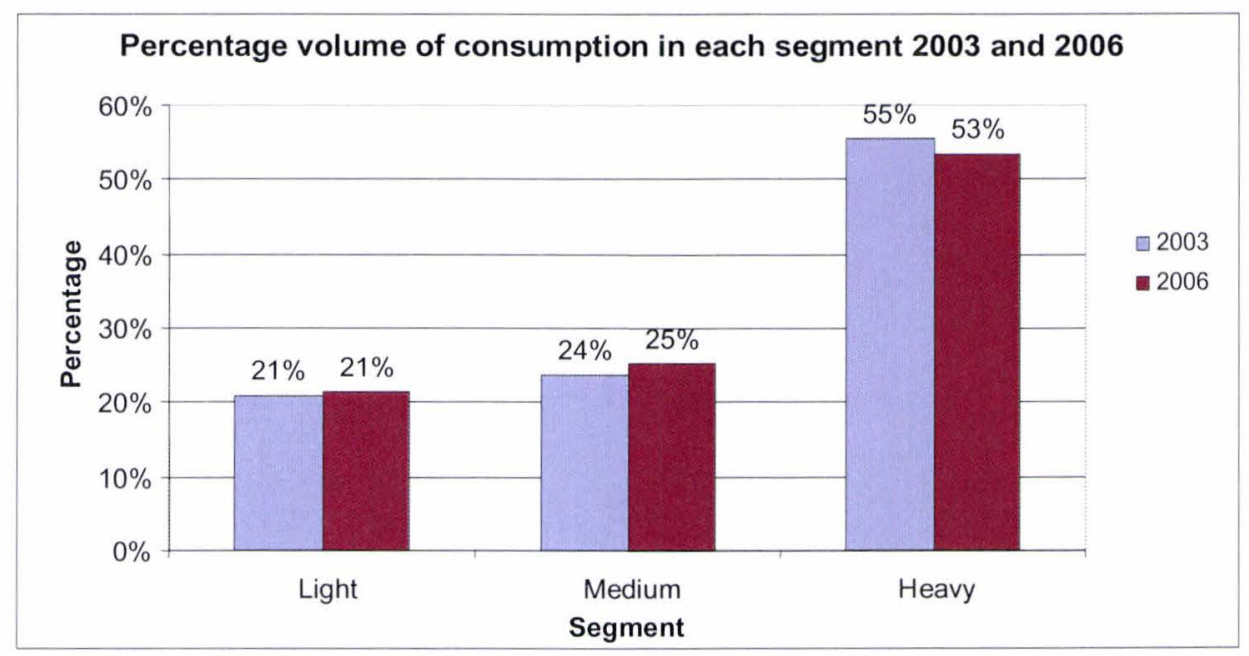


A number of observations are made from the analysis presented in Figure 8 and 9. These observations lead to questioning the merit of using an aggregated approach in customer profiling.

- Rata Energy's largest proportion of customers is in the light segment in 2003 with $44 \%$ (12,330 customers dropping to 1,995 in 2006 - Table 9). In 2006 this profile changes as the heavy segment at $79 \%$ (from 8,951 to 14,197 customers in 2006) dominates the profile. This illustrates the shift from low to high consumption for a large number of the customers over the period of the study.

- The consumption profile in the heavy segment is a different story. It is largely unchanged at the segment level between 2003 and 2006. This is an interesting occurrence when the light segment remained relatively consistent at $21 \%$ consumption proportion, yet lost $33 \%$ of the customers in that segment through to 2006 .

- The medium segment analysis is an interesting profile with customer numbers reducing $15 \%$ and yet consumption remaining high at $31 \%$ from 2003 compared to 2006 .

- The profiles present challenging questions in relation to the behavioural profile and decision-making of these consumers for consumption behaviour and remaining with Rata Energy. In the scenario where lost customers are replaced as a matter of course between 2003 and 2006, profiling customers in this manner 
illustrates how at customer level aggregation leads to a different view of an actual state. This is illustrated in Section 5.7.5 Segment profile 2006.

The somewhat crude application of segmentation in this manner is common and reflects the approach used to date by Rata Energy. The analysis now turns to exploring what actually happens to the customers in the segments we have used so far (light, medium and heavy based on 2003) to achieve greater understanding of the weakness in use of 'point in time' segmentation approach. This is achieved by profiling each of the segments defined in 2003, and comparing the consumption of the actual customers from the 2003 segments in 2006.

\subsubsection{Segment profile 2006}

The analysis below shows the constituency of each 2003 segments and how each group of those customers profiled in 2006. This profile uses categories for those customers who have been lost to Rata Energy as well as the existing consumption categories of light, medium and heavy. 


\subsubsection{Light segment 2003}

Table 10: Light segment of 2003 profiled in 2006

\begin{tabular}{|l|r|r|r|}
\hline \multicolumn{3}{|l|}{ Light segment - customer numbers } \\
\hline & $\mathbf{2 0 0 3}$ & $\mathbf{2 0 0 6}$ & \% in 2006 \\
\hline Lost & - & 6,002 & $49 \%$ \\
\hline Light & 12,330 & 1,424 & $12 \%$ \\
\hline Medium & - & 1,364 & $11 \%$ \\
\hline Heavy & - & 3,540 & $29 \%$ \\
\hline
\end{tabular}

The profile of the light segment in Table 10 changes considerably with only $12 \%$ with this consumption profile in 2006. Of the customers in the light segment in $2003,40 \%$ migrate to higher consumption profile segments with $11 \%$ to medium and $29 \%$ to the heavy segment as seen in Table 10 .

Table 10 also shows $49 \%$ of the customers from the 2003 light segment were no longer customers in 2006. The result of the light segment profiling supports the proposition of Reichheld and Sasser (1990) that longer term customers return more consumption activity, which is the proxy for revenue through cash flow, as only $23 \%$ of the surviving customers from the light segment remain as a light profile customer and the remaining light segment customers increased their consumption. 
Table 11: Medium segment of 2003 profiled in 2006

\begin{tabular}{|c|c|c|c|}
\hline \multicolumn{4}{|c|}{ Medium segment - customer numbers } \\
\hline & 2003 & 2006 & $\%$ in 2006 \\
\hline Lost & - & 2,050 & $30 \%$ \\
\hline Light & - & 271 & $4 \%$ \\
\hline Medium & 6,939 & 225 & $3 \%$ \\
\hline Heavy & - & 4,393 & $63 \%$ \\
\hline
\end{tabular}

There are a number of notable changes in the medium segment profiled in Table 11. $63 \%$ of the original medium segment customers migrate to the heavy segment. A relatively small number of customers remain a medium profile $(3 \%)$, and there is a sizable loss of 30\% customers between 2003 and 2006 and is more than observed in the light category. 
Table 12: Heavy segment of 2003 profiled in 2006

\begin{tabular}{|l|r|r|r|}
\hline \multicolumn{4}{|l|}{ Heavy segment - customer numbers } \\
\hline & $\mathbf{2 0 0 3}$ & $\mathbf{2 0 0 6}$ & \% in 2006 \\
\hline Lost & - & 2,291 & $26 \%$ \\
\hline Light & - & 279 & $3 \%$ \\
\hline Medium & - & 117 & $1 \%$ \\
\hline Heavy & 8,951 & $70 \%$ \\
\hline
\end{tabular}

The heavy segment profile differs from the light and medium segment customer in the customer consumption behaviour. Table 12 shows $70 \%$ of the 2003 customers remaining in this consumption category in 2006. The loss of customers from this category $(26 \%)$ is at a similar scale to that of the medium segment $(30 \%)$ but considerably lower than the light segment (49\%). A notable difference in the segment profile was the low level of customer migration to medium and light consumption segments being $1 \%$ and $3 \%$ to respectively. This level of attrition indicates a tendency for customers to leave in contrast to their consumption profile reducing.

These findings support the propositions of Reichheld and Sasser (1990), Reichheld and Teal (1996) and Berger and Nasr (1998) that longer term customers contribute greater cash flow. As observed in the analysis presented in Tables 8, 9 and 10, the profile of light and medium over time progressed to heavy consumption activity thereby supporting the proposition. Survivors from the light and medium segments showed 
considerable migration activity to heavier segments in 2006 with $40 \%$ (light) and $63 \%$ (medium) customers changing profile.

The heavy and medium segments show relatively the same proportion of lost customers with $26 \%$ from the medium segment and 30\% lost from the heavy segment. The highest loss of customers was in the light segment with $49 \%$.

An area for further investigation is identification of the contributing factors to the loss of customers or the result in change in consumption profile for each segment. The specific reasons for customers leaving Rata Energy are not clear. They may be varied such as dissatisfaction with Rata Energy's service offering, competitive threats, or simply change in lifestyle.

The step of segmenting customers enables us to return to further reliability testing of Berger and Nasr's (1998) CLV calculation model. Undertaking analysis using these smaller groups and inputs has potential to reduce any aggregation bias.

\subsubsection{Re-testing for fit and sensitivity}

The re-testing of Berger and Nasr's (1998) model was undertaken at the segment level. This used the input variables derived from actual defection rates of the first year and not the forecast defections rates based on average previous activity. These were found to be $5 \%$ (Light), $7 \%$ (Medium) and 30\% (Heavy) as outlined in Table 8: Percentage of customers lost each year. This differs from previous analysis in Section 5.4 Model 
calculation compared to actual data, where the model was studied for accuracy in the comparison in results using the retention rate of the base year for the total cohort of customers and not for each of the segments within

The proposition as identified as P6 proposes that this should lead to a closer finding of fit. Table 13 shows that in each instance the model poorly calculated an accurate result. The closest segment was the medium and yet it still had an $8 \%$ variation between the model and the actual CLV calculation. The proposition was that using more granular data (not total cohort average but segments within) then the input variables would have less variation, be more accurate and hence lead to more accurate model calculations. The results of $-12 \%, 8 \%$, and $48 \%$, as observed in Table 13 , showed large variation and hence did not support P6. This result questions the reliability of Berger and Nasr's (1998) model for use in the context of segment and total cohort levels.

Table 13: Fit of model to actual - total cohort and segments

\begin{tabular}{|l|r|r|r|r|r|r|r|}
\hline & $\begin{array}{r}\text { Retention } \\
\text { rate }\end{array}$ & Actual & Model & rifference & $\begin{array}{r}\text { Av. } \\
\text { from } \\
\text { actual }\end{array}$ & $\begin{array}{r}\text { Av. } \\
\text { absolute } \\
\text { error }\end{array}$ & $\begin{array}{r}\text { Std dev } \\
\text { of } \\
\text { difference }\end{array}$ \\
\hline Total cohort & $84 \%$ & $\$ 1,150$ & $\$ 922$ & $\$ 228$ & $20 \%$ & $\$ 507$ & $\$ 649$ \\
\hline Segment & $97 \%$ & $\$ 2,058$ & $\$ 2,313$ & $-\$ 245$ & $-12 \%$ & $\$ 688$ & $\$ 1,094$ \\
\hline Heavy & $93 \%$ & $\$ 1,155$ & $\$ 1,068$ & $\$ 87$ & $8 \%$ & $\$ 360$ & $\$ 416$ \\
\hline Medium & $70 \%$ & $\$ 548$ & $\$ 287$ & $\$ 261$ & $48 \%$ & $\$ 274$ & $\$ 310$ \\
\hline Light &
\end{tabular}




\subsubsection{Is hindsight perfect vision?}

To this point the analysis has been undertaken using the base year only input variables as prescribed by Berger and Nasr (1998) for use when applying their proposed CLV calculation model. The use of segmentation and access to each year's actual data presents opportunity to define the 'actual' defections rates for each year for each segment. This provides the ability to re-run the model using the knowledge of a future state of retention and forms the basis for P7 being that the accuracy of the model would be improved using this level of input data (i.e., would it be more accurate to use the actual retention rate for the four year period than using the 2004 retention rate alone?). The results show disappointing outcomes in relation to improving the models accuracy.

Table 14: Re-calculation based on using an actual average defection rate

\begin{tabular}{|c|c|c|c|c|c|c|c|}
\hline & $\begin{array}{l}\text { Rtn } \\
\text { rate }\end{array}$ & Actual & Model & $\begin{array}{r}\text { Av. } \\
\text { difference }\end{array}$ & $\begin{array}{r}\% \text { from } \\
\text { actual }\end{array}$ & $\begin{array}{r}\text { Av. } \\
\text { absolute } \\
\text { error }\end{array}$ & $\begin{array}{l}\text { Std dev of } \\
\text { difference }\end{array}$ \\
\hline $\begin{array}{l}\text { Total } \\
\text { cohort }\end{array}$ & $86 \%$ & $\$ 1,150$ & $\$ 961$ & $\$ 189$ & $16 \%$ & $\$ 494$ & $\$ 654$ \\
\hline \multicolumn{8}{|l|}{ Segment } \\
\hline Heavy & $91 \%$ & $\$ 2,058$ & $\$ 2,070$ & $-\$ 11$ & $-0.55 \%$ & $\$ 711$ & $\$ 1,029$ \\
\hline Medium & $89 \%$ & $\$ 1,155$ & $\$ 982$ & $\$ 173$ & $15 \%$ & $\$ 398$ & $\$ 425$ \\
\hline Light & $81 \%$ & $\$ 548$ & $\$ 361$ & $\$ 186$ & $34 \%$ & $\$ 274$ & $\$ 285$ \\
\hline
\end{tabular}


Whilst the heavy segment achieved a close fit to the model prediction $(-0.55 \%$ variation), the findings of the other segments were no closer being $15 \%$ for the medium and $34 \%$ for the light segment variation respectively. This is considerable variation and hence implies a lack of accuracy in the model.

The results presented in Table 14, with the exception of the heavy segment group, show considerable variation between actual and calculated results. Consequently, P7 is not supported and puts further doubt on Berger and Nasr's (1998) model's ability to accurately assess a future state.

\subsubsection{Propensity to repurchase (retain)}

Dwyer's (1997) proposition introduced a propensity variable to the repurchase variable to account for the level of risk that a customer is likely to repurchase. However, this is overshadowed by the use of aggregation data for this model as illustrated in the analysis presented in Section 5. Dwyer (1997) focused on non-contractual interactions where purchase recency played a contributing factor in the retention of customers and suggests the issue of recency is not relevant in the contracted context. As such, Dwyer's (1997) contribution to the CLV calculation approach was limited in application as it still carries the risk to accurate calculation identified earlier in this paper of operating with aggregated data. 


\subsection{Contribution}

The research problem identified the opportunity to contribute to CLV calculation theory development by providing empirical evidence to the accuracy and predictive reliability of Berger and Nasr's (1998) CLV calculation model. The need for this research stems from the desire to establish confidence in CLV theory development following papers by Blattberg and Deighton (1996) and Berger and Nasr (1998) and particularly the work relating to the CLV calculation model proposed by Berger and Nasr (1998). The study extends the knowledge of how Berger and Nasr's (1998) CLV calculation model can accurately and reliably forecast the future state of value of a group of customers.

This research has identified a sizable variance when testing for fit between Berger and Nasr's (1998) model forecast and the actual state of customers. The finding of $20 \%$ variance as reported in Table 4 Model vs. actual comparison is considerable and brings into doubt confidence that Berger and Nasr's (1998) CLV model can be relied on to predictively calculate a future state.

The sensitivity of the input variables of retention rate, marketing spend and discount rate were examined. The variable retention rate presented the highest level of sensitivity. The research found the marketing and discount rate variables have little impact on the variability of the value calculation. 
An important finding was the weakness the model has in relation to accommodating the heterogeneity of customers. The use of aggregated data provides a challenge to determining an accurate future state of specific customer segments.

A weakness in Berger and Nasr's (1998) CLV calculation approach is the reliance on a constant retention rate. This presents sizable risk to managers who would use Berger and Nasr's (1998) CLV calculation model outcomes in resource allocation decisions as the model was not capable of reflecting change over the period of a customer relationship. 


\section{Conclusion}

The greater our knowledge increases the more our ignorance unfolds.

John F. Kennedy $(1917-1963)$

It was clear from the literature reviewed on Customer Lifetime Value (CLV) that the foundations for calculation models came from the seminal work of Berger and Nasr (1998). Their CLV model is commonly referred to in subsequent literature in this area, with over two hundred and fifty citations noted on the Google Scholar website. The literature review highlighted that the development of CLV calculation modelling has generally focused on two areas; managerial decision making based on the model results, and the model calculation itself with consideration of the model input variables. The literature highlighted a lack of empirical validation and subsequent testing of the model and related propositions claimed in literature. The assumed accuracy of the model seemed to be unchallenged with a majority of contributions being conceptual in nature.

Highlighted by Reinartz and Kumar (2000) is that Berger and Nasr's (1998) model operates best when applied to situations where customers are in contractual relationships. This was positive as it allowed for examination of Berger and Nasr's (1998) model to the most suited application, being Rata Energy's contracted customer context. Whilst the findings of this research did not support a position that the model calculates accurately the future state of customers, when compared to calculations based on actual data the key area of limitation was identified as the use of the constant 
retention rate and hence the weakness of this models application to non-contractual customer relationships.

The outcome of this research forms an important contribution to academic theory development. This empirical study examined the predictive accuracy of Berger and Nasr's (1998) model and has enabled examination of the affect the input variables have on model calculation result. In addition, seven propositions identified in the literature were examined in the course of this research.

The research has shown considerable variation between the model calculation and the actual data. This presents doubt on the predictive nature of Berger and Nasr's (1998) CLV model. The conclusion is that this may lead managers to poor strategic and resource allocation decisions based on this calculation of value. This has considerable implications on Blattberg and Deighton's (1996) proposed approach to resource allocation and has ongoing impacts on the model development as it may have developed under a wrong assumption of the models accuracy. The variation observed in these findings leads to a lack of confidence in the accuracy of Berger and Nasr's (1998) model given the variation in calculation findings relative to comparing those calculations to actual data. The results also did not indicate any clear bias in the results and will make identification of the model error challenging.

The research also examined input variables of retention rate, marketing spend and discount rate, to provide insight into Berger and Nasr's (1998) model's dynamics. It revealed that the retention rate variable was the most influential of the three input 
variables and as a result the most important input to accurately calculate. The use of a consistent retention rate is flawed and presents a concern as it does not adequately allow for the change in retention over time. Berger and Nasr (1998) suggest their model may be further enhanced through introducing brand loyalty and customer satisfaction variables into the calculation. This may further improve the retention rate input however it does not serve to resolve the issue of variation within an aggregated base or variation of the retention levels over time. Reichheld and Sasser (1990) discussed the challenges for organisations to accurately measure and applying customer satisfaction as an input for buyer behaviour prediction and as such challenged the role this would play in enhancing the model calculation.

The primary conclusion from this research is there is reasonable doubt that Berger and Nasr's (1998) CLV calculation model's can accurately calculate a future value state for a group of Rata Energy residential retail customers. The model does not adequately address customer heterogeneity and is flawed in use of a constant retention rate. Additionally, the model seems relatively insensitive to the input variables particularly the marketing and discount rate inputs. 


\subsection{Summary of findings}

The following is a summarised view of the examined research questions and propositions:

\section{Research question}

Q1

What is the accuracy of the Berger and Nasr's (1998) CLV calculation model to predict a future state (fit - between the model and actual data based calculations)?

The model is not accurate in predicting the future customer lifetime value of the cohort.

The results indicate the model does not have a close fit for purpose. As outlined in Table 4 , the findings of $20 \%$ difference between actual and modelled calculations, is a cause for concern. The resulting conclusion is that managerial decisions such as resource allocation on retention activity must be cautioned if using calculations of this model. 
Q2

How sensitive is the Berger and Nasr's (1998) model calculation output to variation of the input variables?

Little to no impact was observed in testing the sensitivity of the model calculation result to change in each of the input variables. The variable of retention rate was shown to be the most influencing on the CLV calculation outcome.

The CLV model was used to recalculate the value of a customer group by changing each of the input variables to appreciate the level of change needed to affect a $15 \%$ change in the calculation result. Change in the retention variable was found to have a moderate impact on the calculation outcome and was the most influential variable in Berger and Nasr's (1998) CLV model calculations. The calculation outcome was only mildly affected by a change in the marketing spend and discount rate input variables. Tables 5 (retention rate), 6 (marketing spend), and 7 (discount rate) present the results of the sensitivity testing for the three input variables. 


\section{Propositions examined in this study}

P1 Businesses lose 15\% - 20\% of their customers each year

\section{Supported}

The findings of this research support the proposition above. Table 1 reported the average defection rate during the study period was $14 \%$. This finding supports the proposition identified from Reichheld and Sasser (1990). Whilst these findings were on the low side of there proposed attrition rate $(15 \%$ $20 \%$ ) they are found to be sufficient to be supportive of this proposition.

P2 Companies lose half their customers every five years.

\section{Supported}

One approach for calculation is to use the average defection rate of $14 \%$ p.a., if this rate was simply extrapolated over a five year period then $14 \% * 5=70 \%$. This represents a higher rate than suggested in the proposition. Taking a more appropriate approach an average \% change in defection rate is calculated as $17 \%$ to identify what the level of reduction may be in years 4 and 5 . This is based on calculating the average $\%$ reduction over the 3 years (with $6 \%$ reduction between years 1 and 2 and a $27 \%$ reduction between years 2 and 3 
defection rates). Applying this average reduction of $17 \%$ to the years 4 and 5 , the 5 year defection rates are:

- $16 \%$ - actual year 1

- $15 \%$ - actual year 2

- $11 \%$ - actual year 3

- $9 \%$ - calculated year $4(9.13 \%)$

- $8 \%$ - calculated year $5(7.47 \%)$

The sum of these defections is a $59 \%$ defection of customers from the original cohort in 5 years. This result supports the proposition based on the variability of the unknown years 4 and 5. Another recommendation would be to change the proposition statement to include "at least $50 \%$ " to the context of lost customers.

P3 Customers who stay longer increase cash flow over time.

\section{Supported}

The proxy of consumption has been used in this study to represent cash flow. This is based on the assumption that revenue has a direct correlation to the amount a customer purchase/uses.

The finding outlined in Table 3 showed strong growth in the average volume per customer per annum. Customer consumption grew over the four year period from $8,723 \mathrm{kWh}$ in 2003 to $18,502 \mathrm{kWh}$ in 2006 . This demonstrates 
that the flow of revenue (as represented in consumption) does not remain static. This finding shows on average a large increase in volume which would directly translate to revenue for Rata Energy supporting the longer a customer stayed with Rata Energy, revenue increased over those subsequent periods. As such, the proposition is supported. It is acknowledge that this finding is may over simplify the issue in that the growth in consumption may not be due to any aspect of a relationship with Rata Energy and as such acquiring a new customer in later years may show the higher consumption levels immediately. Additionally, other elements need to be factored such as the possible increase in consumers chosen standard of living where electricity consumption increases i.e. people seek warmer houses in winter or use more electronic devices in the home.

P4 Retention rate is the most critical input variable in the CLV calculation.

Supported

Sensitivity analysis was undertaken for each of the input variables identifying that the retention rate had the strongest influence on the model output. Table 5 presents the analysis and shows that to effect a $15 \%$ variation in the CLV model calculation would require $+9 \%$ or a $-10 \%$ change respectively in the retention rate. 
The retention rate had the most influence on the CLV calculation result. The marketing spend and discount rate variables were not found to be as important to the calculation as outlined in the findings presented in Tables 6 and 7.

Acknowledged in this finding was the absence of recorded marketing spend specifically on retention activities by Rata Energy. Rata Energy indicated anecdotally that marketing spend was 'modest' however had no granularity on how marketing spend was allocated. Whilst the sensitivity analysis indicated the model had a low level of impact from change in the marketing spend variable, it is not clear the role Marketing Spend had directly on the size of the retention variable itself prior to calculation. This aside, the actual variable of retention in this calculation was the most influential on the outcome of the calculation.

P5 Different customer segments exhibit different patterns of attrition.

\section{Supported}

The cohort was sorted into three segments (light, medium and heavy) based on consumption in 2003. Analysis found substantial variation in retention rates in each segment, consumption behaviours and activity in relation to defection from Rata Energy or migration to other segments. These findings support the proposition that segments exhibit different characteristics to that of an aggregated profile is supported. 
P6 Using input variables (based on actual 2003 input data) derived at segment level will result in more accuracy in the CLV model calculation.

Not supported

The logic that with more detailed and granular information applied to the model the more accurate the calculation outcome. The outcome of the analysis is presented in Table 13. The results show considerable variation within the cohort at the aggregated level but also between each of the segments.

P7 The use of an average retention rate derived from the actual cohort data will lead to highly accurate results.

\section{Not Supported}

Table 14 presents the findings of the research when using the average retention rate of the cohort over the period. The results revealed no improvement and variation was found in the accuracy of the prediction achieved by the model. 


\subsection{Related observations}

Two areas are discussed to contribute to future insight and discussion on the topic of customer lifetime value calculation.

\subsubsection{The RFM approach}

Recency, frequency and Monetary model introduced by Cullinan (1978) and extended by Baur (1988) is a common methodology used to make segmentation and investment decisions. The analysis and findings presented in this paper have supported the observation of several authors (Fader, Bruce and Berger, 2004 and Kumar, 2006) as articulated by Gupta et al. (2006) "these models [RFM] predict behaviour in the next period only. However, to estimate CLV, we need to estimate customers' purchase behaviour not only in Period 2 but also in Periods 3, 4, 5, and so on" (Gupta et al. 2006, p. 142).

The analysis and findings in this paper indicate there is a level of risk to managerial decisions when using the recency, frequency and monetary value of transactions as the profile of a customer in an early period of a relationship. The analysis conducted in this study showed there was a large change in the make-up of segments based on consumption activity over the study period. Take for example the light consumer group identified in the research. RFM would suggest not investing in these customers and focusing more on the heavy customer groups in Period 2 due to a relatively low consumption and value per customer in Period 1. As was illustrated in Section 5.7 Segment analysis, $49 \%$ of the light segment customers were lost to Rata Energy and 
yet $40 \%$ of that segment in 2003 migrated to larger volume consumer profiles by 2006. Neglecting a growth group of customers based on their 'current profile' in this context would be a poor decision. Further insight into the reasons for customers leaving Rata Energy and for change in their consumption profiles over time will assist further examination of the RFM model however this study has highlighted potential vulnerability in use of the outcome to make resource allocation investment decisions.

\subsubsection{Retention}

The area of risk in customer decision making for purchase was discussed by several authors such as Pfeifer and Carraway (2000) and Rust et al. (2004) who suggested the introduction of Markov Chain Modelling (MCM) to add a probability of customer retention to each year for retention.

Options theory was introduced by Levett et al. (1999) and Rust et al. (2004). Ryals and Knox (2005) introduced a risk adjustment component to the revenue variable. These represent several initiatives by authors to address the challenge of improving predictability of future behaviours and the accuracy of the model. This research has highlighted the challenges of relying too heavily on predictive models that require input assumptions, when the inherent nature of consumer consumption behaviours is variable at all levels, individual, segment and cohort.

The development of model literature has several authors proposing that the CLV calculation can be applied to the valuation of a firm (Gupta and Lehman, 2003; Gupta 
et al., 2004). The model did not produce accurate results when compared to actual data. This was the case for both aggregated and more detailed level segment based application. Therefore there is considerable risk in valuing a business based on this application of the CLV calculation model of Berger and Nasr (1998). 


\section{$7 \quad$ Limitations and future research}

This research has made an important contribution in providing empirical evidence in testing Berger and Nasr's (1998) CLV model accuracy along with examining a number of propositions identified in the review of related academic literature. There were however limitations that are acknowledged in the context of the research findings and several recommendations for future research are presented.

\subsection{Limitations}

\section{Time period}

The period of available data from Rata Energy was limited to four years. Extending a research period would enable deeper insight into the activities associated with the retention rate of the cohort and the segment profiles over time. In addition the period at which a customer was classified as defected was where no consumption was included in that year. This had potential to skew the level of "light" segment customers in one period.

\section{Marketing spend}

The quality of data for defining the marketing spend variable was limited. Ideally, the model should have used the actual spend per customer in 2003 through to 2005 . However, due to no record management at Rata Energy the marketing spend information was only available for 2006. As such, a further study may benefit from 
more specific data inputs. The sensitivity of the marketing spend variable on the model as identified in Section 5.6.3 presents a modest limitation.

\section{Revenue}

The lack of adequate accurate revenue billing data was disappointing, as it would have allowed analysis by volume and value from the billing records. Future research using accurate billing data would enable further testing of propositions, such as Reichheld and Sasser's (1990) on profitability. Given the complexity of the energy retail industry it would still be challenging to accurately identify the marginal revenue of the energy component of billing. This is where empirical analysis of other sectors may provide additional value.

\section{Customer Selection}

Section 5.2.3 Consumption, discussed the potential difference between industry and the cohort's consumption profile. There is the potential that the use of only residential customers in the cohort used for this analysis presents bias when comparisons are made with industry level consumption trends and limited direct comparison. Future analysis may look to present a full spectrum of consumption profiles from business and residential segments.

\subsection{Future research}

Based on the findings of this research, there are several areas to extend the investigations and empirical validation of this subject. This is particularly important 
given the findings of this study where the predictive accuracy of Berger and Nasr's (1998) model was not supported and include:

- Further empirical testing using data from other industry contexts. Identification of the buyer relationship would be valuable i.e. consumer versus household decision making. This will add a level of detail to the findings that is not present from current studies.

- Further empirical studies are suggested to broaden the application of the model to different. Specifically it would be beneficial to apply the model to situations in different categories such as FMCG. This would provide further insight into what results the model produced in different scenarios.

- A based assumption in this research was the treatment of customers. The approach that was adopted was that proposed by Jackson (1985) where we used the context of customers were in the lost-for-good category. Testing consumers in the always-a-share context would add to the robust nature of empirical validation of this model.

- Testing data from a non-contractual transactional context would be beneficial in understanding the accuracy of Berger and Nasr's (1998) CLV model to various scenarios.

- The largest proportion of customers is in the light segment in 2003 however the largest representation is in the heavy segment in 2006. Section 5.7.5 Customer profile identified considerable defection (at least greater than $20 \%$ ) existing in all three segments. Further examination of the reasons for losing the customer 
would provide greater insight into the practical ability for management to reduce the defection.

- Modelling for the optimal level of segmentation is suggested to understand if this will address the issue of customer heterogeneity. Whilst the study grouped customers into three categories, additional segments can be established by adjusting the consumption thresholds. This change would further reduce the size of the customer groups and potentially increase the accuracy of the input variables (specifically retention rate).

- Introducing elements of profitability would add to the findings and insights gained. Cost to serve data would enable examination of the dynamics of profitability as part of understanding CLV better during the relationship with the customer (especially in the context of new electronic channels, which arguably reduce cost to serve when the customer migrates from expensive physical or other channels such as telephone). This also has an implication on the profitability of the customer.

- The treatment of customer acquisition costs is absent and further consideration is recommended when modelling the value of the customer to a firm. As understanding the potential pay-back period for investment in acquisition activity is vital to investment decision making for managers and the lifetime value of that customer.

There are additional considerations that relate specifically to the CLV model and future enhancements: 
- The assumption of a constant probability of retention rate forms a key area for future development and a notable weakness in Berger and Nasr's (1998) CLV model. Establishing an accurate approach to address the future retention rate is vital to improving Berger and Nasr's (1998) CLV model calculation accuracy.

- Broadening the input variables to capture brand, customer satisfaction (Gruca and Rego, 2005) and the competitive context (Berger et al., 2006).

- Addressing the issue of customer heterogeneity is necessary to accurate future application of this model. 


\section{References}

Bauer, C.L. (1988). A direct mail customer purchase model. Journal of Direct Marketing, 2(3), 16-24.

Berger, P.D., Eechambadi, N., George, M., Lehmann, D.R., Rizley, R., and Venkatesan, R. (2006). From customer lifetime value to shareholder value. Theory, empirical evidence, and issues for future research. Journal of Service Research, 9(2), 156-167.

Berger, P.D., and Nasr, N.I. (1998). Customer lifetime value: Marketing models and applications. Journal of Interactive Marketing, 12(1), 17-30.

Berger, P.D., and Nasr-Bechwati, N. (2001). The allocation of promotional budget to maximise customer equity. The International Journal of Management Science, $29,49-61$.

Berger, P.D., Weinberg, B., and Hanna, R.C. (2003). Customer lifetime value determination and strategic implications for a cruise-ship company. Journal of Database Marketing and Customer Strategy Management, 11(1), 40-52.

Blattberg, R.C., and Deighton, J. (1991). Interactive marketing: Exploiting the age of addressability. Sloan Management Review, 33(1), 5-14. 
Blattberg, R.C., and Deighton, J. (1996). Manage marketing by the customer equity test. Harvard Business Review, July-August.

Blattberg, R.C., Getz, G., and Thomas, J.S. (2001). Customer equity: Building and managing relationships as valuable assets. Boston: Harvard Business School Press.

Colombo, R. and Jiang, W. (1999). A stochastic RFM model. Journal of Interactive Marketing, 13(3), Summer, 2-12.

Cullinan, G.J. (1978). Picking them by their batting averages: Recency-frequencymonetary. Method of Controlling Circulation. Manual Release. New York: Direct Mail/Marketing Association.

Dowling, G.R. and Uncles, M. (1997). Do Customer Loyalty Programs Really Work? Sloan Management Review, 38, Summer, 71-82.

Dickson, P.L. (1997). Marketing management, (2nd ed.). Orlando, F1: Harcourt Brace College Publisher.

Dwyer, R.F. (1997). Customer lifetime valuation to support marketing decision making. Journal of Direct Marketing, 11(4), Fall, 6-13. 
Fader, P.S., Bruce, G.S. and Berger, P.D. (2004). Customer base analysis with discrete-time transaction data. Unpublished working paper.

Gronroos, C. (1991). The marketing strategy continuum: A marketing concept for the 1990s. Management Decision, 29(1), 7-13.

Gruca, T.S., and Rego, L.L. (2005). Customer satisfaction, cash flow and shareholder value. Journal of Marketing, 69 115-130.

Gupta, S., Lehmann, D.R. (2003). Customers as assets, Journal of Interactive Marketing, 17(1), 9-24.

Gupta, S., Lehmann, D.R., and Stuart, J.A. (2004). Valuing customers. Journal of Marketing Research. (2), 7-18.

Haenlein, M., Kaplan, M.A., and Schoder, D. (2006). Valuing the real option of abandoning unprofitable customers when calculating customer lifetime value. Journal of Marketing, 70 (July), 5-20.

Hayashi, F. (1982). Toblin's marginal $q$ and average $q$. A neoclassical interpretation. Econometrica, 59, (January), 213-224.

Helm, S. (2003). Calculating the value of customers' referrals. Managing Service Quality, 13(2), 124-133. 
Jackson, B.B. (1985). Winning and keeping industrial customers. Lexington, MA, Lexington Books.

Jain, D., and Singh. S.S. (2002). Customer lifetime value research in marketing: A review and future directions. Journal of Interactive Marketing, 16(2), 34-46.

Kotler, P. (1997). Marketing management. Analysis, planning, implementation, and control. Englewood Cliffs, NJ: Prentice-Hall, Inc.

Kotler, P., and Armstrong, G. (1996). Principals of marketing, ( $7^{\text {th }}$ ed.). Englewood Cliffs, NH: Prentice-Hall, Inc.

Kumar, V. (2006). CLV: A path to higher profitability. Working paper, University of Connecticut, Storrs.

Kumar, V., Ramani, G., and Bohling, T. (2004). Customer lifetime value approaches and best practice applications. Journal of Interactive Marketing, 15(3), 60-72.

Levitt, T. (1986). The marketing imagination. New York: MacMillian, Inc.

Levett, P., Page, M., Nel, M.P., Pitt, L., Berthon, P., and Money, A. (1999). Towards an application of option pricing theory in the valuation of customer relationships. Journal of Strategic Marketing, 7, 275-284. 
Libai, B., Narayandas, D., and Humby, C. (2002). Toward an individual customer profitability model - A segment-based approach. Journal of Service Research, $5(1), 69-76$.

McKenna, R. (1991). Relationship marketing. London: Addison Wesley Publishing Firm.

McKenna, R. (1993). Relationship marketing: Successful strategies for the age of the consumer. Boston: Harvard Business School Press.

Miglautsch, J. (2002). Application of RFM principles: What to do with 1-1-1 customers? Journal of Database Marketing, 9(4), 319-325.

Morgan, R.M., and Hunt, S.D. (1994). The commitment trust theory of relationship marketing. Journal of Marketing, 58 (July), 30-37.

Mulhern, F.J. (1999). Customer profitability analysis: Measurement, concentration, and research directions. Journal of Interactive Marketing, 13(1), 25-40.

Pfeifer, P.E., and Carraway, R.L. (2000). Modelling customer relationships as Markov chains, Journal of Interactive Marketing, 14(2), 43-55. 
Pfeifer, P.E., Haskkins, M.E., and Conroy, R.M. (2004). Customer lifetime value, customer profitability, and the treatment of acquisition spending. Journal of Managerial Issues, April, 1-25.

Rata Energy. (2007). Options choices decisions - Understanding the options for making decisions about New Zealand's electricity future. Wellington, New Zealand: Rata Energy.

Reichheld, F.F. (1996). Learning from customer defections. Harvard Business Review, March, 56-69.

Reichheld, F.F., and Sasser, W.E. Jnr.. (1990). Zero defections: Quality comes to services. Harvard Business Review, September-October, 105-111.

Reichheld, F.F., and Teal, T. (1996). The loyalty effect. Boston: Harvard Business School Press.

Reinartz, W.J., and Kumar, V. (2000). On the profitability of long-life customers in a non-contractual setting: An empirical investigation and implications for marketing. Journal of Marketing, 64 17-35.

Reinartz, W.J., and Kumar, V. (2003). The impact of customer relationship characteristics on profitable lifetime duration. Journal of Marketing, 67(1), 7799. 
Reinartz, W.J., Thomas, J.S., and Kumar, V. (2005). Balancing acquisition and retention resources to maximise customer profitability. Journal of Marketing, 69 (January), 63-79.

Rust, R.T., Lemon, K.N., and Zeithaml, V.A. (2004). Return on marketing: Using customer equity to focus marketing strategy. Journal of Marketing, 68 (January), 109-127.

Ryals, L.J., and Knox, S. (2005). Measuring risk-adjusted customer lifetime value and its impact on relationship marketing strategies and shareholder value. European Journal of Marketing, 39(5/6), 456-472.

Schmittlein, D.G., Morrison, D.G., and Colombo, R. (1987). Counting your customers: Who are they and what will they do next? Management Science, 33 (January), 124.

Schmittlein, D.G., and Peterson, R.A. (1994). Customer base analysis: An industrial purchase process application. Marketing Science, 13 (Winter), p. 41-67.

Sharp, B. (2005). A 5\% drop in defections does not result in $80 \%+$ increase in profits. Retrieved July 2008 from http://www.marketingscience.info/. Ehrenberg-Bass Institute for Marketing Science, University of South Australia.

Shepherd, D. (1990). The new direct marketing. Homewood, IL: Business One Irwin. 
Shih, Y.Y., and Liu, C.Y. (2003). A method for customer lifetime value ranking Combining he analytic hierarchy process and clustering analysis. Journal of Database Marketing and Customer Strategy Management, 11(2), 159-173.

Stone, B. (1995). Successful direct marketing methods. Lincolnwood, IL: NTC Business Books.

Van Raaji, E.M. (2005). The strategic value of customer profitability analysis. Marketing Intelligence and Planning, 23(4), 372-381.

Van Triest, S. (2005). Customer size and customer profitability in non-contractual relationships. Journal of Business and Industrial Marketing, 20(3), 148-155.

Webster, F.E. (1994). Market-driven management. New York: John Wiley and Sons, Inc.

Wyner, G.A. (1996). Customer profitability: Linking behaviour to economics. Marketing Research, 8(2), p. 36-38.

Wyner, G.A. (1999). Customer Relationship Management. Marketing Research: A Magazine of Management and Applications, 11(2), 39-41. 